\title{
Petrological and Geochemical Constraints in the Origin and Associated Mineralization of A-Type Granite Suite of the Dhiran Area, Northwestern Peninsular India
}

\author{
L. Gopeshwor Singh ${ }^{1, *}$, G. Vallinayagam ${ }^{2}$ \\ ${ }^{1}$ National Institute of Rock Mechanics, Kolar Gold Fields-563117, India \\ ${ }^{2}$ Department of Geology, Kurukshetra University, Kurukshetra-136 119, India
}

\begin{abstract}
Neoproterzoic, anorogenic, A-type granites of Dhiran area of Malani Igneous Suites is made up wholly of peralkaline-peraluminous alkali feldspar granites and is composed of K-feldspar, quartz, alkali amphibole, plagioclase, biotite and accessory minerals are iron oxides, monozite, zircon, apatite, and annite. This granitoid is associated with acid volcanic and minor basic volcanic-plutonic and dykes. Petrographically, they show cloudy, patchy perthitic texture and graphic texture. They are highly evolved ferroan, alkaline, A-type granites, displaying the typical geochemical characteristics of A-type granites with high $\mathrm{SiO}_{2}, \mathrm{Na}_{2} \mathrm{O}+\mathrm{K}_{2} \mathrm{O}, \mathrm{FeO} * \mathrm{MgO}, \mathrm{Ga} / \mathrm{Al}, \mathrm{Zr}, \mathrm{Nb}, \mathrm{Ga}, \mathrm{Y}, \mathrm{Ce}$ and rare earth elements (REE) and low $\mathrm{CaO}, \mathrm{MgO}, \mathrm{Ba}$ and $\mathrm{Sr}$. Their trace and REE characteristics along with the use of various discrimination schemes revealed their correspondence to magmas derived from crustal origin. The extremely high $\mathrm{Rb} / \mathrm{Sr}$ ratios combined with the obvious $\mathrm{Sr}$, $\mathrm{Ba}, \mathrm{P}, \mathrm{Ti}$ and Eu depletions clearly indicate that these A-type granites were highly evolved and require advanced fractional crystallization in upper crustal conditions. These granites are high to medium content of radioactive element (U, Th, K) together Heat generation and Heat Production (HP). Leucocratic granites show higher Heat Production $\left(4.84-8.75 \mu \mathrm{Wm}^{-3}\right)$ and total Heat Generation Unit (11.52-20.84 HGU) than pink granite (2.09-2.94 $\mu \mathrm{Wm}^{-3}$ (HP), 4.98-7.07 HGU). The granites of the Dhiran area show higher average value of total Heat Generation Unit (16.51 HGU and 5.79 HGU) than the average value of $3.8 \mathrm{HGU}$ for the continental crust. The high Heat Generation Unit values (4.98-20.84) of the Dhiran granites indicate 'hot crust' category and a possible linear relationship between the surface heat flow and crustal heat generation in the Malani Igneous Suite.
\end{abstract}

Keywords A-type, Alkaline, Anorogenic, Mineralization, Malani Igneous Suite

\section{Introduction}

Anorogenic, A-type granites are characterized by high $\mathrm{SiO}_{2}, \mathrm{Na}_{2} \mathrm{O}+\mathrm{K}_{2} \mathrm{O}, \mathrm{Fe} / \mathrm{Mg}, \mathrm{Ga} / \mathrm{Al}, \mathrm{Rb}, \mathrm{Nb}, \mathrm{Zr}, \mathrm{Ta}, \mathrm{Y}, \mathrm{Cs}, \mathrm{Ga}$, $\mathrm{U}, \mathrm{Th}, \mathrm{REE}$ (except $\mathrm{Eu}$ ) and low abundances of $\mathrm{MgO}, \mathrm{CaO}$, $\mathrm{Mg}^{\#}, \mathrm{Ba}, \mathrm{Sr}, \mathrm{P}, \mathrm{Ti}, \mathrm{Ni}, \mathrm{Cr}, \mathrm{Co}, \mathrm{V}([1,2])$. The presence of one or more of such ferromagnesian minerals as annite rich biotite, ferrohastingsite, alkali amphibole and $\mathrm{Na}$-pyroxene $([3,4])$ are typical for A-type granites. Many models have been proposed to the origin of A-type granitoids includes: 1. remelting of previously melted granulite source rock containing quartz - alkali feldspar - plagioclase $([1,2,5]) ; 2$. partial melting of dehydrated charnockitic lower crust which formed as a residue from the earlier I-type magma, at temperature $>900^{\circ} \mathrm{C}$ in a subduction-related tectonic setting $[4]$; 3. dehydration melting of calc-alkaline

* Corresponding author:

gopenirm@gmail.com (L. Gopeshwor Singh)

Published online at http://journal.sapub.org/geo

Copyright (C) 2012 Scientific \& Academic Publishing. All Rights Reserved granitoids $([6,7]) ; 4$. differentiation from mantle-derived basaltic magma[8,9]; 5. dehydration melting of amphibolebearing tonalite at 6-10 Kbar leaving behind a granulitic residue, produce melts that resembles A-type granite, except for their somewhat high $\mathrm{Al}_{2} \mathrm{O}_{3}$ contents[10]; 6. small degree of partial melting of a felsic infracrustal source region, with water and $\mathrm{F}$ contents similar to those recorded in I-type granitoids source region[11]. The remelting residual source idea has been questioned([4,7,10,12]). Creasers et al.,[7] argued that residual source melting could not produce the characteristic major elements composition of A-type granites. Anderson,[6] suggested partial melting of tonalitic to granodiorite crust to form A-type granitic melts. Furthermore, Skjerlic and Johnston ([10,12]) suggested subsolidus dhydroxylation (OH-F) increase the stability of biotite in the lower crust to $>950^{\circ} \mathrm{C}$ which upon melting causes $\mathrm{F}$ enrichment in the magma. On the other hands, Landenberger and Collins[4] suggested that the halogens (F, Cl) are not a major factor in A-type granite formation. A-type melts are considered to be anhydrous on account of characteristic occurrence of interstitial amphibole and/or biotite $([1,2])$. 
The anhydrous nature of A-type granite, low abundance of $\mathrm{Rb}$ and fairly high $\mathrm{Sr}$ contents contradict the formation of A-type melts by anhydrous mineral fractionation from I-type magma[1].

A-type granites or alkaline, peralkaline and metaluminous-peralumi nous granitoids constitute a considerable volume of the Malani Igneous Suits (MIS) and they have attracted the interest of several researchers, indicating their important in crust formation and cratonization of the shield. Granitoids with chemical and mineralogical characteristics of A-type or within-plate granites as define for instance by Collins et al.,[1], Whalen et al.,[2] and Eby,[3] are quite common in the MIS. These intrusive are considered to represent anorogenic, A-type magma generated in rift related environment of high heat flow and abundant volatile activity, correlative with an extensional tectonic regime[13] and probably including melts generated from both upper mantle and lower crustal source. The purposed of present communication is to provide a detailed petrological and geochemical data on the A-type granite and associated mineralization around Dhiran, so as to infer their petrogenesis and tectonic setting.

\section{Regional Geological Aspects}

Malani Igneous Suits (MIS) is unique in the geological evolution of the Western Indian Shield as it is characterized by a major period of anorogenic, A-type, within plate, high heat producing (HHP) magmatism[14-20]. The Neoproterozoic MIS $\left(55,000 \mathrm{Km}^{2} ; 750 \pm 10 \mathrm{Ma}\right)$ comprising peralkaline (Siwana) metaluminous to mildly peralkaline (Jalor) and peraluminous (Tusham and Jhunjhunu) granites with cogenetic carapace of acid volcanic (welded tuff, trachyte explosion braccia and perlite) are characterized by volcano-plutonic ring structure and redial dykes. The suits is bimodal in nature with minor amounts of basalts, gabbro and dolerite dykes.

The Neoproterozoic magmatism in Western Peninsular India during Delhi Orogenesis led to the formation of intrusive Syntectonic Erinpura granite/ granodiorite (900 Ma)[21]. It is followed by late-tectonic granitoid plutonism of Mount Abu batholiths $(800 \pm 50 \mathrm{Ma})$. The continuum of this acid magmatism culminated with outpour of bimodal volcanism and emplacement of A-type granites of MIS[22]. The MIS consists predominantly of acid volcano-plutonic rocks that are seen as scattered outcrops to the West of the Palaeoproterozoic to Mesoproterozoic Aravalli and Delhi Supergroup. Rocks of MIS are underlain by metasediments/granitoids of the Mesoproterozoic Delhi Supergroup and unconformably overlain either by Pokharan boulder bed of glacial origin of Vendian age (680 to $580 \mathrm{Ma}$ ) or by sediments of Marwar Supergroup (Vendian to Lower Cambrian)[23]. Kochhar,[24] established a close association in space and time between the explosive acid lava and high-level granite that form distinct ring structure and radial dyke. The volcanic-plutonic rocks association of MIS has three episodes of igneous activity[25], the first episode represent by widespread acid and basic lava flows covering about $31,000 \mathrm{sq}$. $\mathrm{km}$. of MIS. It was followed by a major plutonic activity that comprises discordant plutons, bosses and ring dykes of granite whereas the last stage includes emplacement of acid and basic dyke swarms. The preponderance of acid volcanic rocks relative to intermediate and basic rocks is a distinctive feature of MIS.

Dhiran area is located between longitude $72^{\circ} 17^{\prime} \mathrm{E}$ to $72^{\circ}$ $30^{\prime} \mathrm{E}$ and latitude $25^{\circ} 27^{\prime} \mathrm{N}$ to $25^{\circ} 31^{\prime} \mathrm{N}$ in the Northwestern Peninsular India. The area is predominantly occupied (90\%) by rhyolite rocks. The area is characterized by A-type granite with cogenetic carapace of acid volcanics and with minor amount of basalt, gabbro and dolerite dyke (Fig. 1). Based on detailed geological mapping and position of xenoliths, Dhiran area is established that extrusive phase (trachyte, rhyolite, welded tuff, basalt) was followed by the intrusive phase (alkali granite) and the magmatic activity was culminated by the dyke phase (Dolerite, rhyolite, microgranite).

The granites are leucocratic, pink in colour, fine to medium grained and show incrustation of iron oxide. The dark pink medium grained granite show altered (cloudy appearance) alkali feldspar with white rim, pink tint (alteration of orthoclase). The cloudiness of alkali feldspar has been reported in many Scottish Caledonian intrusive[26] and in Nigeria Younger Granite[27] as well as from the granites of Dhiran area in MIS which illustrates the post - magmatic fluid interaction. The cross-cutting of granites by numerous quartz veins with iron oxides strain or fluorine incrustation, aplite knots and numerous pegmatite veins indicates the presence of aqueous fluids that are responsible for enrichment of rare metals[28]. Close association of felsic volcanic rocks (rhyolite, trachyte) with granites, mafic volcanic (basalt) and plutonic (gabbro) rocks indicates an interrelationship between the volcanism and plutonism.

\section{Petrography}

The pink granite consist essential of quartz, K-feldspar (perthite, orthoclase), arfvedsonite, aegirine, riebeckite, aenigmatite, plagioclase and accessory minerals are hematite, ilminite, zircon, annite, apatite, monazite and rutile. Whereas leucocratic granite contains quartz, K-feldspar (perthite, microcline), albite, biotite, muscovite as major phase and minor phase are apatite, zircon, magnetite, hematite, ilmenite and annite. Hypidiomorphic, granophyric and microgranophyric textures are preserved in these granites. Quartz shows wavy extinction and occurs as micro-granophyric intergrowth and in the groundmass. Perthites are characterized by cloudy, patchy, incoherent and extensive coarsening nature due to feldspar-fluid interaction at subsolidus temperature $([29,30])$ that leads to the replacement of albite at the margin of the perthite, which appears as whitish on turbid portions at the crystal margins or along cracks and cleavages. At place, minute mica flakes are also observable along the margins of perthite as post-magmatic phases due to accumulation of residual fluid. Orthoclase is medium grained, subhedral and shows carlsbad twinning. The arfvedsonite is prismatic in 
shape, dark green coloured and pleochroic $(X=$ dark bluish green; $\mathrm{Y}=$ bluish green; $\mathrm{Z}=$ yellowish green) with an extinction angle $\left(\mathrm{X}^{\wedge} \mathrm{C}\right)$ of $13-15^{\circ}$. Short prismatic aegirine shows pleochroism $(\mathrm{X}=$ dark green, $\mathrm{Y}=$ light green, $\mathrm{Z}=$ yellow green) with an extinction angle $\left(X^{\wedge} C\right) 4-7^{\circ}$. Whereas riebeckite shows pleochroism ( $\mathrm{X}=$ light blue: $\mathrm{Y}=$ blue: $\mathrm{Z}=$ dark blue) with an extinction angle $\left(\mathrm{X}^{\wedge} \mathrm{C}\right)$ of $3-5^{\circ}$. Subhedral crystals of bright green colour aegirine are also present along the perthite with groundmass. Albite occurs as lath shaped crystal that exhibit polysynthetic twinning. Biotite is strongly pleochroic $(\mathrm{X}=$ yellowish brown: $\mathrm{Y}=$ reddish brown: $\mathrm{Z}=$ olive green) with corroded and partly or fully resorbed. It also contains pleochrole haloes around minute zircon crystals. Magnetite occurs as anhedral isotropic mineral in the groundmass and inclusion in perthite and quartz. Hematite is subhedral and reddish brown in colour whereas ilmenite is fine grained and silver grey in colour. Both hematite and ilmenite occur in feldspar phenocrysts and in the groundmass as well. Monozite occurs as light yellow fine crystal in the groundmass. In the alkali feldspar-quartz-plagioclase feldspar diagram (Fig. 2), both the granites (leucocratic granite and pink granite) are shown as alkali feldspar granite in nature.

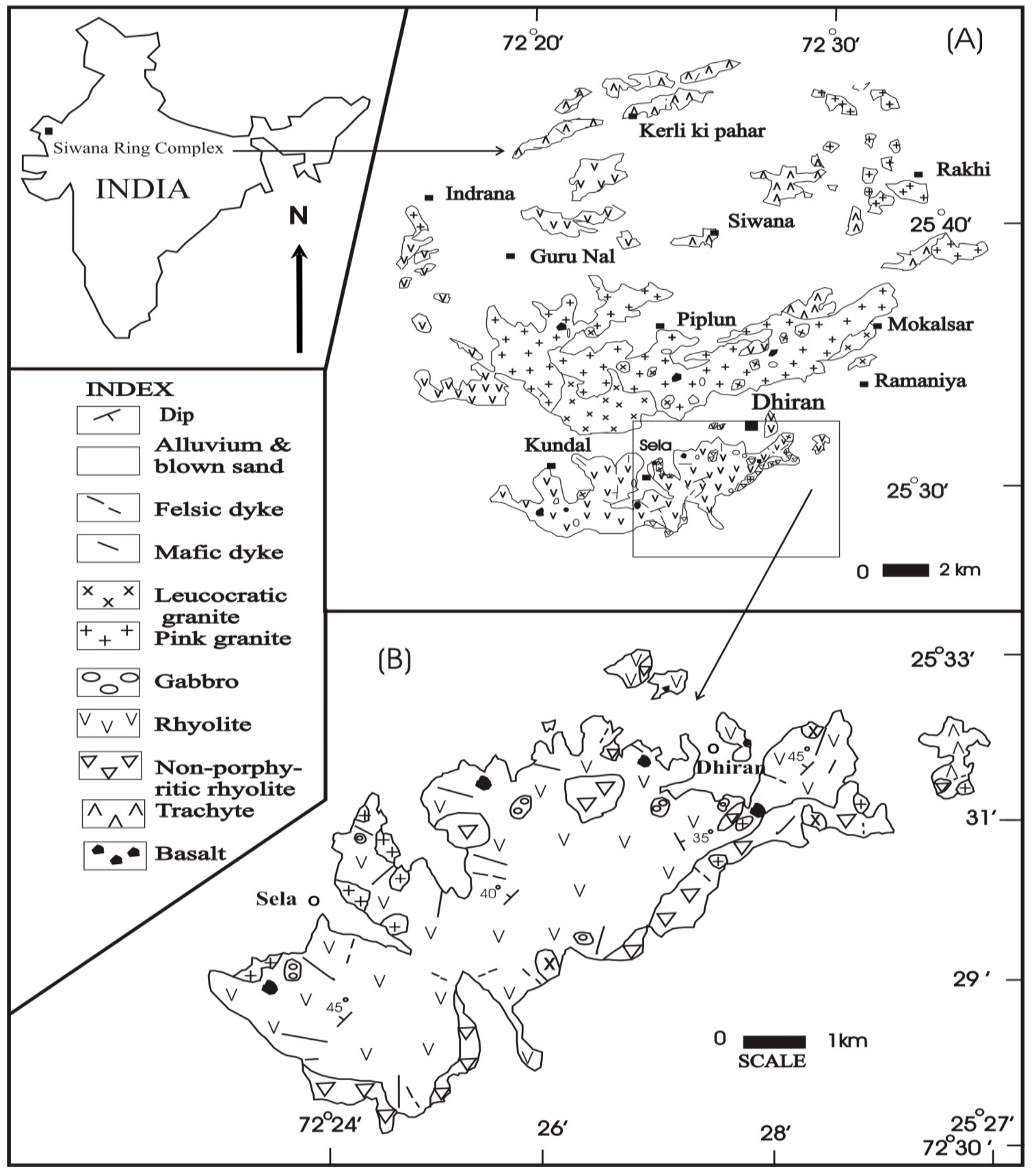

Figure 1. A. Geological Map of Siwana Ring Complex, Malani Igneous Suites (MIS). B. Simplified lithological Map of Dhiran Area 


\begin{tabular}{|l|}
\hline Key to the QAP triangle \\
2 Alkali feldspar granite \\
3a Syenogranite \\
3b Monzogranite \\
4 Granodiorite \\
5 Tonalite \\
6* Quartz alkali feldspar syenite \\
6 Alkali feldspar syenite \\
7* Quartz syenite \\
7 Syenite \\
8* Quartz monzonite \\
Monzonite \\
9* Quartz monzodiorite/ \\
Quartz monzogabbro \\
9 Monzodiorite/Monzogabbro \\
10* Quartz diorite/Quartzgabbro/ \\
$\quad$ Quartz anorthosite \\
10 Diorite/Gabbro/Anorthosite \\
Key to the Tectonic Boundaries \\
POG=Post-orogenic granitoids \\
CCG=Continental collision granitoids \\
CAG= Continental arc granitoids \\
IAG = Island arc granitoids \\
RRG= Rift-related granitoids \\
CEUG=Continental epirogenic uplift granitoids \\
\hline
\end{tabular}

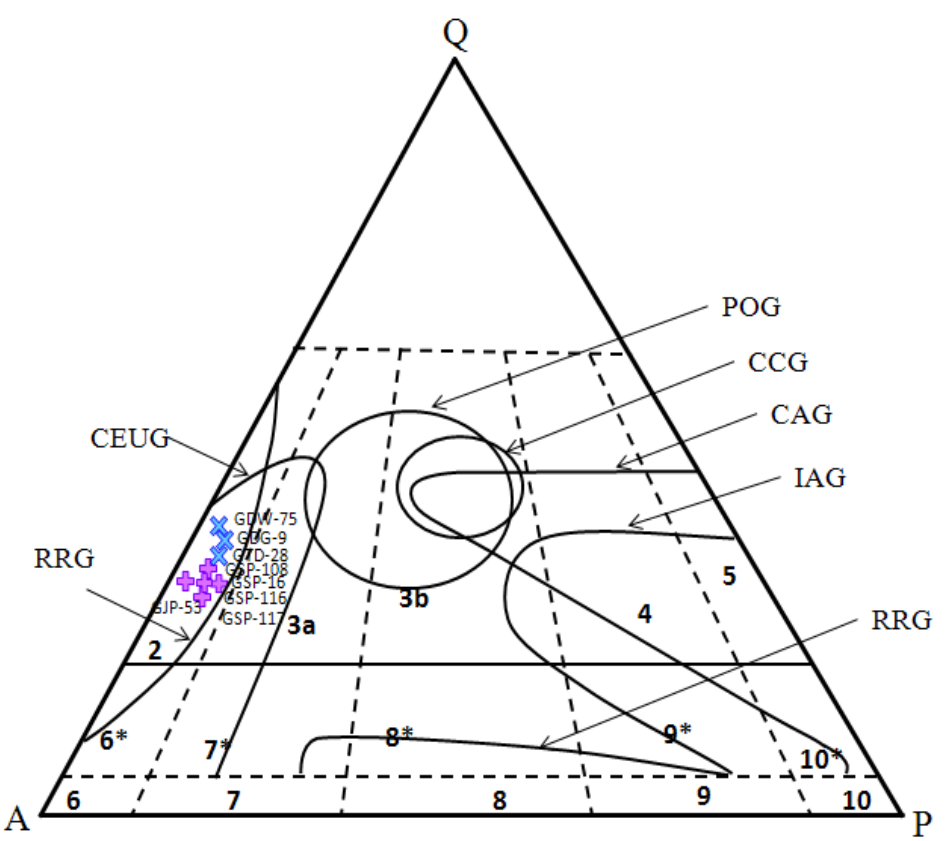

Figure 2. Plots of granites samples on the QAP diagram[31]. Zones of tectonic environments are after Maniar and Piccoli[32]. Symbols: Pink granite $\left({ }^{\text {f5 }}\right)$ and Leucocratic granite $(æ)$

Moreover, among the tectonic discrimination boundaries adopted by Maniar and Piccoli,[32] on the QAP diagram, they fall in the Anorogenic granites field rift-related granitoids (RRG) and continental epirogenic uplift granitoids (CEUG).

\section{Geochemistry}

The rocks chemistry of major, trace and REE elements analyses was carried out on Atomic Absorption Spectrophotometry (AAS), Department of Geology, Kurukshetra University Kurukshetra, Inductively Coupled Plasma Atomic Emission Spectrometry (ICP-AES), School of Environmental Sciences Jawaharlal Nehru University, New Delhi and Inductively Coupled Plasma Emission Massspectrometry (ICP-MS), National Geophysical Research Institute (NGRI), Hyderabad. The discrimination and correlation diagram are plotted to characterize each granite type and to discuss the petrogenesis and mineralization of the granites from the study area.

\subsection{Major, Trace and REE Element Classification}

The result of the chemical analyses are given in the tables 1A, 1B and 1C. Both the granite samples from the Dhiran area are remarkable homogeneous with respect to both major and trace element geochemistry. The analysed rocks show a restricted range in chemical composition. The concentration of $\mathrm{SiO}_{2}$ in the Pink granite are low $\mathrm{SiO}_{2}(65.85-69.90)$ and high total iron (5.48-8.39) as compared to leucocratic granites: $\mathrm{SiO}_{2}$ (73.89-77.74) and total iron (1.10-5.06). The average values of $\mathrm{Al}_{2} \mathrm{O}_{3}, \mathrm{TiO}_{2}, \mathrm{Na}_{2} \mathrm{O}, \mathrm{K}_{2} \mathrm{O}, \mathrm{CaO}, \mathrm{MgO}$ and $\mathrm{P}_{2} \mathrm{O}_{5}$ of the pink granites are $12.49,0.79,4.48,4.96,1.02$, 0.27 and 0.20 which are comparatively same as Leucocratic granites: $12.19,0.26,3.60,4.02,0.71,0.12$ and 0.01 respectively. Both the granites ranges from peralkaline[(acmite normative $>1)$; Agpaitic Index ( $\mathrm{AI}=$ molecular portion of $\left.\mathrm{Na}_{2} \mathrm{O}+\mathrm{K}_{2} \mathrm{O} / \mathrm{Al}_{2} \mathrm{O}_{3}\right)$ and alumina saturation index $(\mathrm{A} / \mathrm{CNK}=$ molecular $\left.\mathrm{Al}_{2} \mathrm{O}_{3} / \mathrm{Na}_{2} \mathrm{O}+\mathrm{K}_{2} \mathrm{O}+\mathrm{CaO}\right)$ ratio $<1$ ] peraluminous (corundum and anorthite normative ; $\mathrm{AI}>1$ ) (Fig. 3). Further, the widely used $\mathrm{SiO}_{2}$ vs $\mathrm{K}_{2} \mathrm{O}$ diagram[33] classify most of the samples as high-K rocks, with exceptional few samples belong to the shoshonite series (Fig. 4). Their very low contents of $\mathrm{CaO}, \mathrm{MgO}$ with $\mathrm{Fe} / \mathrm{Mg}$ ratios and $\mathrm{AI}$ values signify alkali affinity of these granites[34]. These magmatic rocks attained very high content of sodium and iron as well as strongly depleted in alumina along with calcium and magnesium in their final stages of magmatic and subsolidus processes. Hence, the nature of these low alumina A-type rocks appeared to have been formed under the control of alkali-alumina relationship as evident from this textural and mineralogical data $([35,36])$. Dhiran peralkaline and peraluminous granites plots in the normative of $\mathrm{Qz}-\mathrm{Ab}-\mathrm{Or}$ ternary diagram[6]. In this diagram (Fig. 5) peralkaline granites falls varies $5 \mathrm{~kb}$ to more than $10 \mathrm{~kb}$ pressure and peraluminous granites lies vary $<2 \mathrm{~kb}$ to $4 \mathrm{~kb}$ pressure. It is suggested that the peralkaline granites are emplaced at greater depth $\left(16-35 \mathrm{~km}\right.$ and $\left.480^{\circ} \mathrm{C}-840^{\circ} \mathrm{C}\right)$ and more fluorine content. Thus, Dhiran peralkaline granites are generated at a greater depth than the peraluminous granite. The geochemical nature of the studied A-type granites are critically tested using the standard common schemes as well as the recently adopted three-tiered geochemical classification scheme of granites rocks[37]. As the extreme $\mathrm{Fe}^{*} \mathrm{O}$ enrichment relative to $\mathrm{MgO}$ (high $\mathrm{FeO}^{*} / \mathrm{MgO}$ ) is a typical signature of A-type granitoids (Fig. 6), all the present granite samples are grouped as ferroan A-type granite (Fig. 7) and on the $\mathrm{Fe}^{*}\left\{\mathrm{FeO}^{*} /(\mathrm{FeO}+\mathrm{MgO})\right\}$ vs $\mathrm{SiO}_{2}$ diagram[37]. 

of A-Type Granite Suite of the Dhiran Area, Northwestern Peninsular India

Table 1A. A. Major elements concentration and CIPW norms of the Dhiran granites, Malani Igneous Suite, Northwestern Peninsular India

\begin{tabular}{|c|c|c|c|c|c|c|c|c|c|c|c|c|c|c|}
\hline Rock Type & \multicolumn{9}{|c|}{ Pink Granite } & \multicolumn{5}{|c|}{ Leucocratic Granite } \\
\hline $\begin{array}{c}\text { Sample } \\
\text { No. }\end{array}$ & $\begin{array}{c}\text { GSP1 } \\
7\end{array}$ & $\begin{array}{c}\text { GSP1 } \\
\text { 08 }\end{array}$ & $\begin{array}{c}\text { GSP1 } \\
16\end{array}$ & $\begin{array}{c}\text { GSP1 } \\
17\end{array}$ & $\begin{array}{c}\text { GTP1 } \\
07\end{array}$ & $\begin{array}{c}\text { GTP1 } \\
09\end{array}$ & $\begin{array}{c}\text { GTP1 } \\
10\end{array}$ & $\begin{array}{c}\text { GJP } \\
53\end{array}$ & $\begin{array}{c}\text { GKP1 } \\
11\end{array}$ & $\begin{array}{l}\text { GD } \\
\text { G8 }\end{array}$ & $\begin{array}{c}\text { GWD } \\
75\end{array}$ & $\begin{array}{c}\text { GDG } \\
10\end{array}$ & $\begin{array}{c}\text { GPT } \\
28\end{array}$ & $\begin{array}{c}\text { GDP } \\
29\end{array}$ \\
\hline \multicolumn{15}{|c|}{ Major elements (wt.\%) } \\
\hline $\mathrm{SiO}_{2}$ & 67.07 & 68.69 & 68.01 & 65.85 & 66.84 & 67.42 & 69.90 & 67.81 & 69.32 & 74.17 & 73.89 & 74.02 & 76.32 & 77.74 \\
\hline $\mathrm{TiO}_{2}$ & 0.96 & 0.62 & 0.68 & 0.83 & 0.88 & 0.96 & 0.69 & 0.93 & 0.58 & 0.39 & 0.30 & 0.40 & 0.13 & 0.16 \\
\hline $\mathrm{Al}_{2} \mathrm{O}_{3}$ & 13.12 & 12.04 & 11.55 & 13.01 & 12.14 & 12.52 & 12.11 & 12.93 & 13.04 & 12.75 & 13.59 & 12.54 & 10.67 & 11.40 \\
\hline $\mathrm{FeO}^{*}$ & 6.53 & 8.39 & 7.65 & 7.74 & 7.59 & 5.48 & 5.03 & 6.96 & 5.57 & 5.06 & 2.72 & 3.62 & 1.10 & 2.44 \\
\hline $\mathrm{MnO}$ & 0.24 & 0.13 & 0.30 & 0.14 & 0.17 & 0.19 & 0.17 & 0.18 & 0.08 & 0.07 & 0.17 & 0.09 & 0.01 & 0.01 \\
\hline $\mathrm{MgO}$ & 0.12 & 0.36 & 0.31 & 0.24 & 0.20 & 0.24 & 0.12 & 0.27 & 0.63 & 0.02 & 0.18 & 0.05 & 0.19 & 0.16 \\
\hline $\mathrm{CaO}$ & 0.85 & 0.35 & 1.02 & 0.67 & 1.15 & 1.40 & 1.58 & 0.78 & 1.46 & 0.59 & 0.68 & 0.80 & 0.71 & 0.78 \\
\hline $\mathrm{Na}_{2} \mathrm{O}$ & 4.80 & 3.99 & 4.54 & 4.35 & 4.66 & 5.09 & 4.46 & 4.30 & 4.20 & 3.29 & 3.35 & 3.64 & 4.10 & 3.66 \\
\hline $\mathrm{K}_{2} \mathrm{O}$ & 5.45 & 4.45 & 4.67 & 5.14 & 4.76 & 5.62 & 4.65 & 5.36 & 4.56 & 3.52 & 3.69 & 4.04 & 5.17 & 3.32 \\
\hline $\mathrm{P}_{2} \mathrm{O}_{5}$ & 0.18 & 0.01 & 0.18 & 0.12 & 0.17 & 0.22 & 0.45 & 0.14 & 0.35 & 0.01 & 0.01 & 0.01 & 0.01 & 0.01 \\
\hline $\mathrm{H}_{2} \mathrm{O}$ & 0.54 & 1.16 & 1.18 & 1.09 & 0.98 & 0.67 & 0.91 & 0.41 & 0.25 & 0.12 & 0.35 & 0.38 & 0.42 & 0.23 \\
\hline Total & 99.86 & 99.69 & 99.79 & 99.18 & 99.54 & 99.81 & 100.07 & 100.08 & 100.04 & 99.99 & 98.93 & 99.59 & 98.83 & 99.91 \\
\hline Quartz & 18.25 & 27.19 & 23.58 & 19.80 & 21.03 & 7.04 & 25.23 & 21.21 & 26.73 & 40.30 & 38.59 & 35.63 & 35.34 & 41.86 \\
\hline Orthoclase & 32.21 & 26.30 & 27.60 & 30.37 & 28.13 & 33.21 & 27.48 & 31.67 & 26.95 & 20.80 & 21.81 & 23.87 & 30.55 & 19.62 \\
\hline Albite & 37.14 & 33.76 & 33.41 & 36.81 & 35.94 & 33.11 & 36.40 & 36.39 & 35.54 & 27.84 & 28.35 & 30.80 & 26.10 & 30.97 \\
\hline Anorthite & -- & 1.67 & -- & 0.79 & -- & -- & -- & 0.15 & 3.26 & 2.86 & 3.31 & 3.90 & -- & 3.80 \\
\hline Corundom & -- & 0.05 & -- & -- & -- & -- & -- & -- & -- & 2.48 & 2.87 & 0.75 & -- & 0.39 \\
\hline Acmite & 3.06 & -- & 4.41 & -- & 3.07 & 8.77 & 1.18 & -- & -- & -- & -- & -- & 3.18 & -- \\
\hline Diopsite & 0.50 & -- & 1.67 & -- & 1.07 & 1.29 & 0.64 & 0.21 & -- & -- & -- & -- & 1.02 & -- \\
\hline $\begin{array}{l}\text { Hyper- } \\
\text { sthene }\end{array}$ & 0.07 & 0.90 & -- & 0.60 & -- & -- & -- & 0.57 & 1.57 & 0.05 & 0.45 & 0.12 & 0.27 & 0.40 \\
\hline $\begin{array}{c}\text { Wollas- } \\
\text { tonite }\end{array}$ & -- & -- & 0.73 & -- & 0.34 & 0.52 & 0.97 & -- & -- & -- & -- & -- & 0.72 & -- \\
\hline Magnetite & -- & -- & 0.46 & -- & -- & -- & -- & -- & -- & -- & -- & -- & -- & -- \\
\hline Illmenite & 0.51 & 0.06 & 0.34 & 0.30 & 0.36 & 0.41 & 0.36 & 0.39 & 0.17 & 0.15 & 0.36 & 0.19 & 0.02 & 0.02 \\
\hline Hematite & 5.47 & 8.39 & 5.81 & 7.74 & 6.53 & 2.45 & 4.62 & 6.96 & 3.57 & 5.56 & 2.72 & 3.62 & -- & 2.44 \\
\hline Apatite & 0.42 & 0.02 & 0.42 & 0.28 & 0.39 & 0.51 & 1.04 & 0.32 & 0.81 & 0.02 & 0.02 & 0.02 & 0.02 & 0.02 \\
\hline Sphene & 1.69 & -- & -- & 1.23 & 1.69 & 1.83 & 1.22 & 1.78 & 1.20 & -- & -- & -- & 0.29 & -- \\
\hline Rutite & -- & 0.11 & -- & 0.22 & -- & -- & -- & -- & -- & 0.40 & 0.14 & 0.38 & -- & 0.19 \\
\hline DI & 87.60 & 87.25 & 84.59 & 86.98 & 85.10 & 83.36 & 89.11 & 89.27 & 89.22 & 88.94 & 88.75 & 90.30 & 91.94 & 92.45 \\
\hline $\mathrm{AI}$ & 1.05 & 0.95 & 1.08 & 0.98 & 1.06 & 1.07 & 1.02 & 0.99 & 0.91 & 0.72 & 0.69 & 0.83 & 1.15 & 0.84 \\
\hline$(\mathrm{Na}+\mathrm{K})$ & 10.25 & 8.44 & 9.21 & 9.49 & 9.42 & 10.71 & 9.11 & 9.66 & 8.76 & 6.81 & 7.04 & 7.68 & 9.27 & 6.98 \\
\hline$(\mathrm{Na} / \mathrm{K})$ & 0.88 & 0.89 & 0.97 & 0.85 & 0.98 & 0.91 & 0.95 & 0.80 & 0.91 & 0.93 & 0.90 & 0.90 & 0.79 & 1.10 \\
\hline $\mathrm{KN} / \mathrm{C}$ & 12.05 & 24.35 & 9.02 & 14.16 & 8.19 & 7.65 & 5.76 & 12.38 & 6.00 & 11.54 & 10.53 & 9.60 & 13.79 & 8.95 \\
\hline A/CNK & 0.84 & 1.00 & 0.80 & 0.93 & 0.81 & 0.74 & 0.79 & 0.90 & 0.89 & 1.23 & 1.27 & 1.06 & 0.78 & 1.04 \\
\hline $\mathrm{An} / \mathrm{An}+\mathrm{Ab}$ & -- & 0.04 & -- & 0.02 & -- & -- & -- & 0.01 & 0.08 & 0.09 & 0.10 & 0.11 & -- & 0.10 \\
\hline
\end{tabular}

Table 1B. Trace and REE concentration of granites from Dhiran Area, Malani Igneous Suite Northwestern Peninsular India

\begin{tabular}{|c|c|c|c|c|c|c|}
\hline \multicolumn{4}{|c|}{ Pink Granite } & \multicolumn{3}{|c|}{ Leucocratic Granite } \\
\hline SAMPLE & GSP17 & GTP109 & GJP53 & GWD75 & GDG10 & GDT28 \\
\hline $\mathrm{Sc}$ & 4.499 & 5.94 & 4.86 & 3.603 & 1.591 & 1.693 \\
\hline $\mathrm{V}$ & 4.246 & 2.509 & 10.518 & 7.324 & 4.129 & 3.829 \\
\hline $\mathrm{Cr}$ & 1.07 & 1.335 & 2.289 & 50.308 & 2.061 & 3.203 \\
\hline Co & 1.71 & 1.258 & 2.838 & 0.564 & 0.396 & 0.327 \\
\hline $\mathrm{Ni}$ & 0.38 & 0.31 & 1.871 & 1.377 & 1.462 & 1.21 \\
\hline $\mathrm{Cu}$ & 0.478 & 0.313 & 0.692 & 0.561 & 0.534 & 0.465 \\
\hline $\mathrm{Zn}$ & 47.988 & 44.01 & 22.455 & 19.403 & 27.334 & 11.601 \\
\hline $\mathrm{Ga}$ & 57.603 & 46.878 & 40.135 & 39.51 & 39.86 & 22.398 \\
\hline $\mathrm{Rb}$ & 92.557 & 115.618 & 89.317 & 411.307 & 319.025 & 250.622 \\
\hline $\mathrm{Sr}$ & 17.262 & 19.781 & 37.922 & 19.193 & 12.758 & 15.599 \\
\hline $\mathrm{Y}$ & 259.235 & 217.849 & 236.226 & 542.545 & 365.974 & 164.131 \\
\hline $\mathrm{Zr}$ & 1181.96 & 1138.81 & 3064.338 & 6568.279 & 4355.924 & 312.666 \\
\hline $\mathrm{Nb}$ & 80.465 & 81.744 & 95.523 & 156.255 & 108.241 & 29.768 \\
\hline Cs & 1.198 & 7.767 & 0.672 & 2.468 & 2.314 & 12.956 \\
\hline $\mathrm{Ba}$ & 233.473 & 278.078 & 161.361 & 29.933 & 27.625 & 35.999 \\
\hline $\mathrm{Hf}$ & 33.603 & 31.346 & 35.927 & 79.683 & 56.781 & 11.574 \\
\hline $\mathrm{Ta}$ & 3.881 & 3.935 & 5.699 & 2.031 & 5.486 & 2.198 \\
\hline $\mathrm{Pb}$ & 11.298 & 17.244 & 30.646 & 131.22 & 49.939 & 34.164 \\
\hline Th & 11.925 & 11.381 & 18.123 & 67.623 & 51.145 & 31.286 \\
\hline $\mathrm{U}$ & 3.603 & 3.16 & 4.75 & 13.829 & 12.249 & 3.475 \\
\hline $\mathrm{La}$ & 106.598 & 110.455 & 140.097 & 261.698 & 226.782 & 128.431 \\
\hline $\mathrm{Ce}$ & 281.117 & 262.634 & 344.405 & 690.733 & 488.914 & 147.259 \\
\hline $\operatorname{Pr}$ & 35.407 & 33.381 & 42.594 & 66.802 & 59.642 & 32.815 \\
\hline $\mathrm{Nd}$ & 157.301 & 145.115 & 186.342 & 270.023 & 242.028 & 139.736 \\
\hline Sm & 37.423 & 32.399 & 41.793 & 62.493 & 52.559 & 31.709 \\
\hline $\mathrm{Eu}$ & 4.816 & 3.696 & 3.095 & 2.492 & 2.066 & 0.492 \\
\hline $\mathrm{Gd}$ & 32.961 & 28.569 & 34.171 & 53.611 & 44.287 & 25.387 \\
\hline $\mathrm{Tb}$ & 6.331 & 5.443 & 6.252 & 10.851 & 8.335 & 4.404 \\
\hline Dy & 44.781 & 38.031 & 43.901 & 81.941 & 61.49 & 29.691 \\
\hline Ho & 5.262 & 4.388 & 4.802 & 9.479 & 7.033 & 3.252 \\
\hline $\mathrm{Er}$ & 17.304 & 14.667 & 16.169 & 33.176 & 24.076 & 10.982 \\
\hline $\mathrm{Tm}$ & 2.273 & 1.897 & 2.147 & 4.529 & 3.206 & 1.445 \\
\hline
\end{tabular}


Table 1C. Radioelements, Heat Production and radioactive Heat Generation Unit data of granites from Dhiran area, Malani Igneous Suites, Northwestern Peninsular India

\begin{tabular}{|c|c|c|c|c|c|c|c|c|c|c|}
\hline Sample No & $\mathrm{U}(\mathrm{ppm})$ & Th (ppm) & K (\%) & $\mathrm{Th} / \mathrm{U}$ & Ur & $\begin{array}{c}\text { Heat Ge } \\
\mathrm{U}\end{array}$ & $\begin{array}{l}\text { ion (HC } \\
\text { Th }\end{array}$ & $\begin{array}{l}\text { due to } \\
\mathrm{K}\end{array}$ & $\begin{array}{l}\text { Total } \\
\text { HGU } \\
\end{array}$ & $\mathrm{HP}\left(\mu \mathrm{Wm}^{-3}\right)$ \\
\hline GWD75 & 13.829 & 67.62 & 3.06 & 4.89 & 53.76 & 8.61 & 11.52 & 0.7 & 20.84 & 8.75 \\
\hline GDG10 & 12.25 & 51.15 & 3.65 & 4.18 & 45.12 & 7.63 & 8.71 & 0.84 & 17.18 & 7.22 \\
\hline GDT28 & 8.47 & 31.29 & 4.3 & 3.75 & 32.59 & 5.26 & 5.33 & 0.99 & 11.52 & 4.84 \\
\hline GSP17 & 3.603 & 11.93 & 4.52 & 3.13 & 18.61 & 2.24 & 2.03 & 1.04 & 5.32 & 2.23 \\
\hline GTP109 & 3.16 & 11.38 & 4.67 & 3.6 & 18.19 & 1.97 & 1.94 & 1.07 & 4.98 & 2.09 \\
\hline GJP53 & 4.75 & 18.12 & 4.44 & 3.82 & 22.69 & 2.96 & 3.09 & 1.02 & 7.07 & 2.97 \\
\hline
\end{tabular}

$\mathrm{Ur}=$ Radioelement concentration; $\mathrm{HP}=$ Heat Production; HGU $=$ Heat Generation Unit

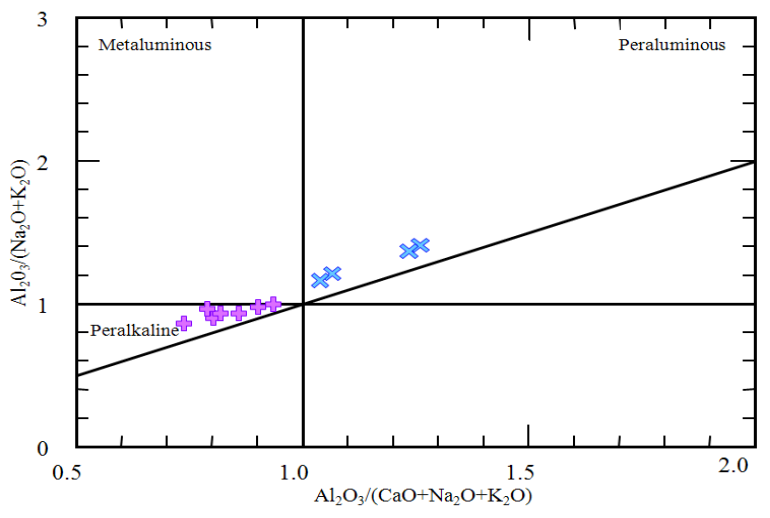

Figure 3. $\mathrm{Al}_{2} \mathrm{O}_{3} /\left(\mathrm{CaO}+\mathrm{Na}_{2} \mathrm{O}+\mathrm{K}_{2} \mathrm{O}\right)$ vs $\mathrm{Al}_{2} \mathrm{O}_{3} /\left(\mathrm{Na}_{2} \mathrm{O}+\mathrm{K}_{2} \mathrm{O}\right)$ diagram $[32]$ showing the fields of Dhiran granites

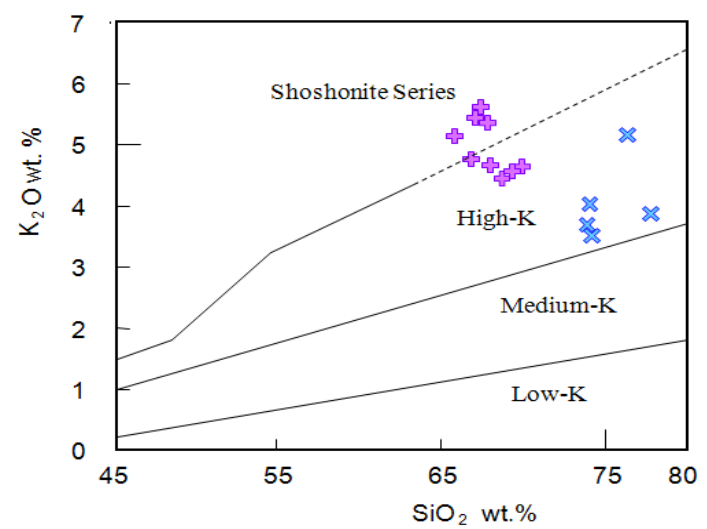

Figure 4. $\mathrm{SiO}_{2}$ vs $\mathrm{K}_{2} \mathrm{O}$ diagram with field after Rickwood[33]

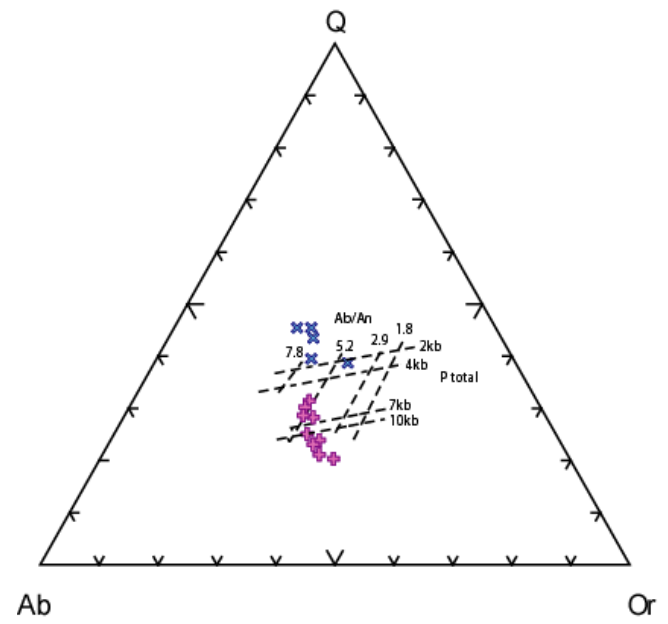

Figure 5. Normative Quartz - Albite - Orthoclase of Dhiran granites and composition to experimental minimum melt composition. Minimum melt experimental grid after Anderson,[6]
Projection of our samples on Frost et al.[37] modified alkali-lime index $\left(\mathrm{Na}_{2} \mathrm{O}+\mathrm{K}_{2} \mathrm{O}-\mathrm{CaO}\right)$ vs. $\mathrm{SiO}_{2}$ diagram plot, the pink granites are in the alkalic field and leucocratic granites are in the alkali-calcic field (Fig. 8). Because of granitoids can contrast from corundum normative $\left(\mathrm{Al}_{2} \mathrm{O}_{3}\right.$ oversaturated) to acmite normative $\left(\mathrm{Al}_{2} \mathrm{O}_{3}\right.$ critically undersaturated), alumina saturation appears to be the most important criterion in classification of granitoids. Fig. 3 reveals that the studied A-type granites are peralkaline to peraluminous. Peralkaline samples are acmite and $\mathrm{Na}_{2} \mathrm{SiO}_{3}$ normative and have $\mathrm{A} / \mathrm{CNK}$ values less than one. Peraluminous A-type granites are corundum normative and have $\mathrm{A} / \mathrm{CNK}$ ratios greater than one. The major and trace elements geochemistry of igneous rocks bear a close relationship to their tectonic setting of formation.

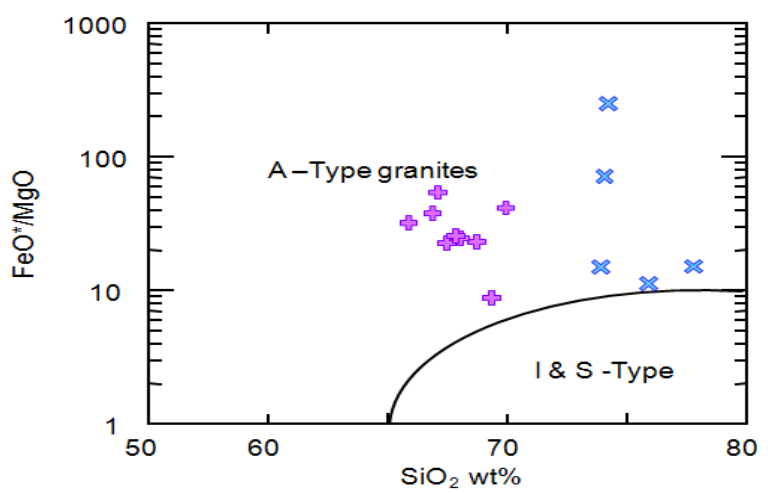

Figure 6. Plots of Dhiran granites on $\mathrm{SiO}_{2}$ vs $\mathrm{FeO} * / \mathrm{MgO}$ diagram[3]. Note that A-type granites are characterized by higher $\mathrm{Fe} * / \mathrm{MgO}$ ratios compared with other granitoid types

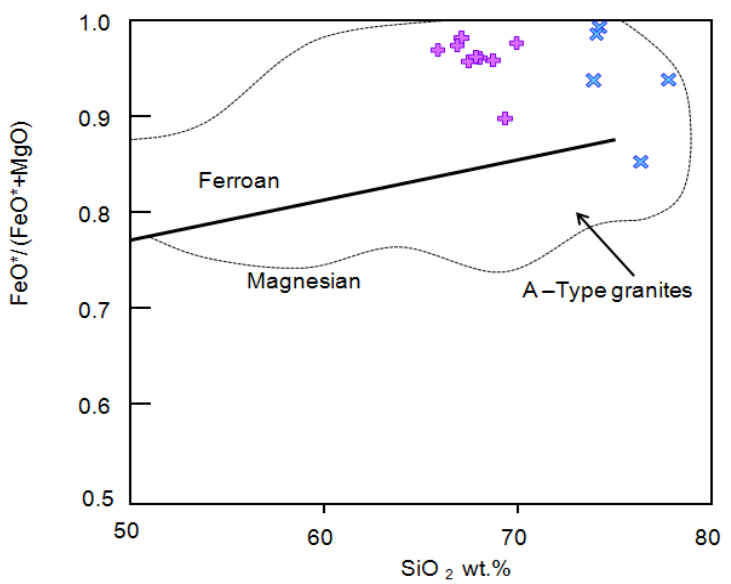

Figure 7. Chemical classification of Dhiran granites using $\mathrm{FeO} * /(\mathrm{FeO}+\mathrm{MgO})$ vs $\mathrm{SiO}_{2}$ diagram 
According to Maniar and Piccoli's,[32] granitoids tectonic classification scheme based on major elements $\left(\mathrm{SiO}_{2}\right.$ vs $\mathrm{Al}_{2} \mathrm{O}_{3}$ diagram (Fig. 9), the studied A-type granitoid rocks are classified as anorogenic granites (CEUG and RRG). All the sample are assigned to a within-plate tectonic environment (Fig. 10). In the R1 - R2 diagram (Fig. 10) majority of the samples are also plotted in the anorogenic field, suggesting their emplacement in a within-plate tectonic setting. The prominent peralkaline and alkaline nature of the studied granitoids are consistent with their affiliation to extensional environments[38]. The $\mathrm{Rb} / \mathrm{Sr}$ vs $\mathrm{K} / \mathrm{Rb}$ diagram (Fig. 11) was found to be a useful discriminent between orogenic and anorogenic granites[39]. According to this diagram Dhiran granites are plots in the anorogenic field. Use of the popular Pearce et al.,[40] trace element discrimination diagrams (Fig. 12 and Fig. 13), for tectonic interpretation of granites rocks ( $\mathrm{Y}$ vs Nb and $\mathrm{Y}+\mathrm{Nb}$ vs $\mathrm{Rb}$ ), all samples were plotted in both diagrams in WPG, as well in the field of A-type granites in the $\mathrm{Y}$ vs $\mathrm{Nb}$ diagram, delineated by Stern and Gottfried[41]. These granites are low in $\mathrm{Mg} \#\left(\mathrm{Mg} / \mathrm{Mg}+\mathrm{Fe}^{\mathrm{t}}\right.$ cation mole percent), $\mathrm{MgO}, \mathrm{FeO}^{*}, \mathrm{CaO}, \mathrm{Sr}$ and high $\mathrm{SiO}_{2}, \mathrm{Na}_{2} \mathrm{O}, \mathrm{K}_{2} \mathrm{O}$, $\mathrm{Fe} / \mathrm{Mg}, \mathrm{Y}$ and show both the LREE and HREE enriched patterns with large negative $\mathrm{Eu}$ anomalies as common in A-type granitiods $([1,2,43])$.

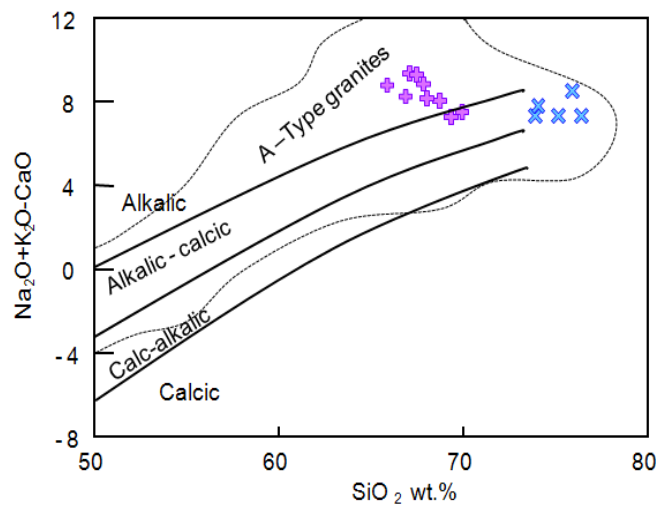

Figure 8. Chemical classification of Dhiran granites using $\mathrm{Na}_{2} \mathrm{O}+\mathrm{K}_{2} \mathrm{O}-\mathrm{CaO}$ vs $\mathrm{SiO}_{2}$ diagram

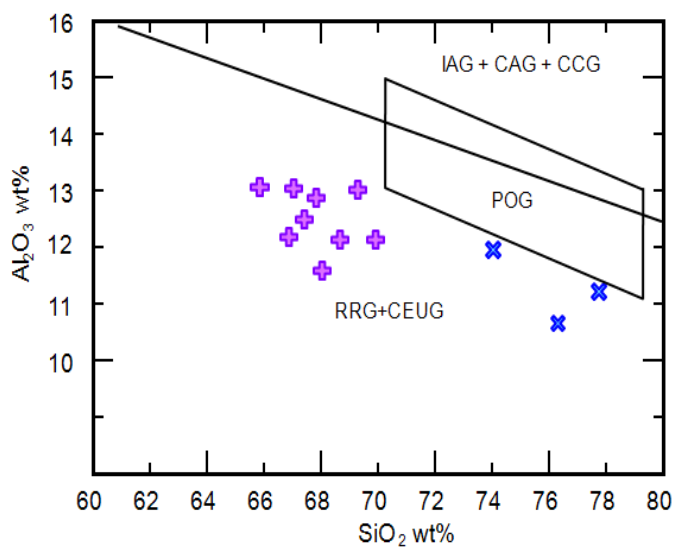

Figure 9. $\mathrm{SiO}_{2}$ vs $\mathrm{Al}_{2} \mathrm{O}_{3}$ diagram[32]. Dhiran granites plots in the RRG + CEUG field. $I A G=$ Island arc granitoids, $C A G=$ Continental arc granitoids, $\mathrm{CCG}=$ Continental collision granitoids, $\mathrm{POG}=$ Post orogenic granitoids, $\mathrm{RRG}=$ Rift related granitoids and $\mathrm{CEUG}=$ Continental Epeirogenic uplift granitoids

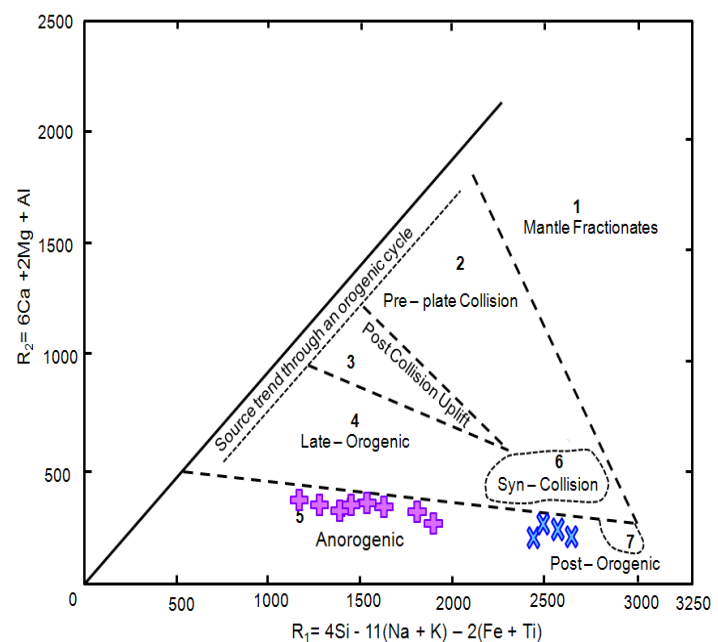

Figure 10. $\mathrm{R}_{1}-\mathrm{R}_{2} 4 \mathrm{Si}-11(\mathrm{Na}+\mathrm{K})-2(\mathrm{Fe}+\mathrm{Ti})$ vs $\left.(6 \mathrm{Ca}+2 \mathrm{Mg}+\mathrm{Al})\right]$ Diagram[42]

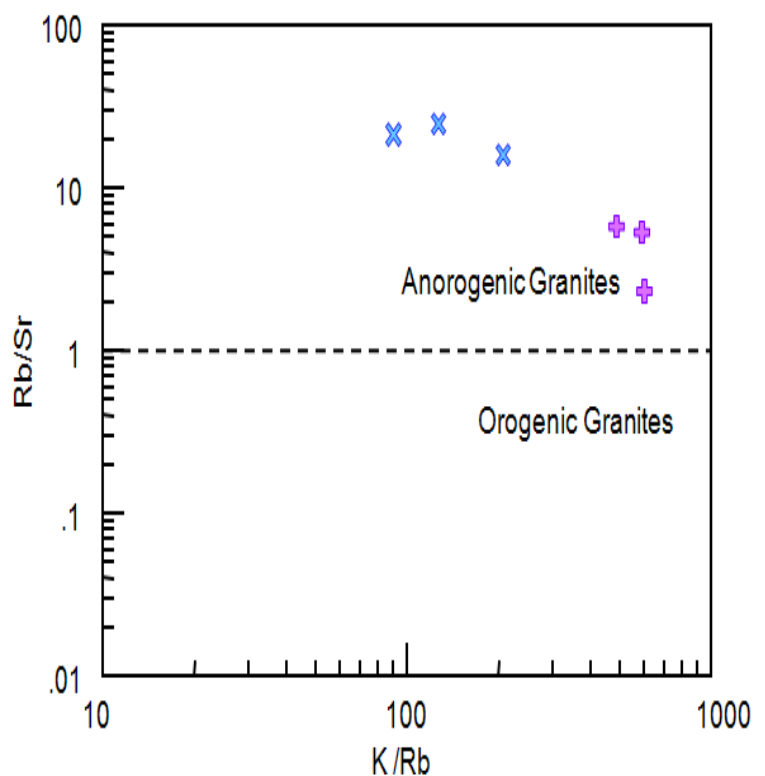

Figure 11. $\mathrm{K} / \mathrm{Rb}$ vs. $\mathrm{Rb} / \mathrm{Sr}$ diagram showing that all the data points of the present A-type granites exhibit $\mathrm{Rb} / \mathrm{Sr}$ ratios higher than one similar to anorogenic granites, whereas the orogenic calc-alkaline granites are characterized by lower ratios[39]

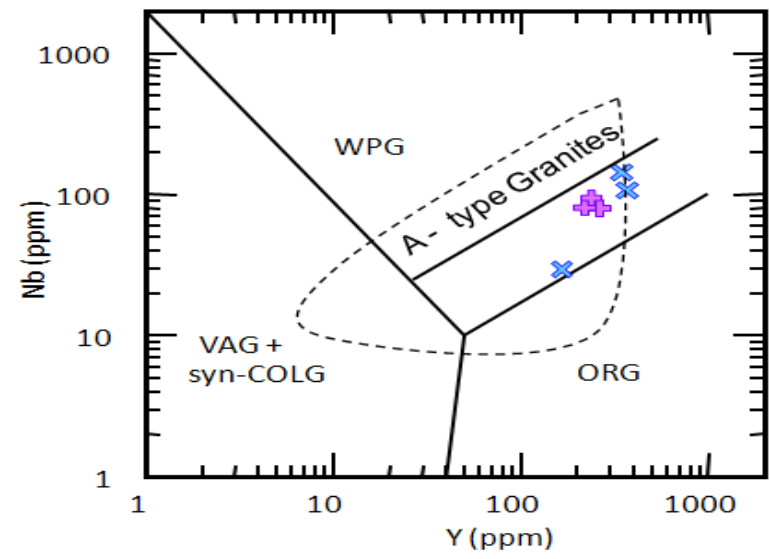

Figure 12. Plots of Dhiran granites on the tectonic discrimination diagram $\mathrm{Nb}$ vs $\mathrm{Y}$ of Pearce et al.,[40]. Field of A-type granites in the $\mathrm{Nb}$ vs $\mathrm{Y}$ diagram is after Stern and Gottfried,[41] 


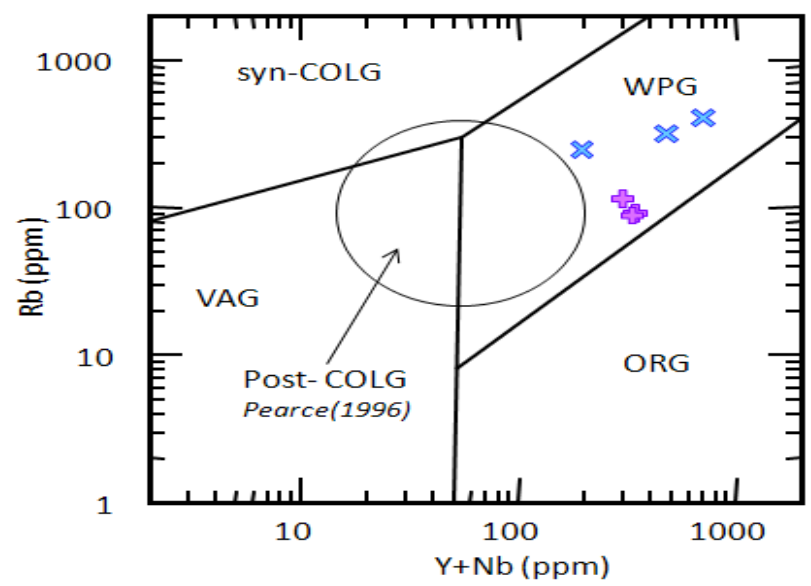

Figure 13. Plots of Dhiran granites on the tectonic discrimination diagram $\mathrm{Rb}$ vs $(\mathrm{Y}+\mathrm{Nb})$ of Pearce et al.,[40]. Field of post-COLG in the Rb vs $(\mathrm{Y}+\mathrm{Nb})$ diagram is adopted by Pearce, [52]

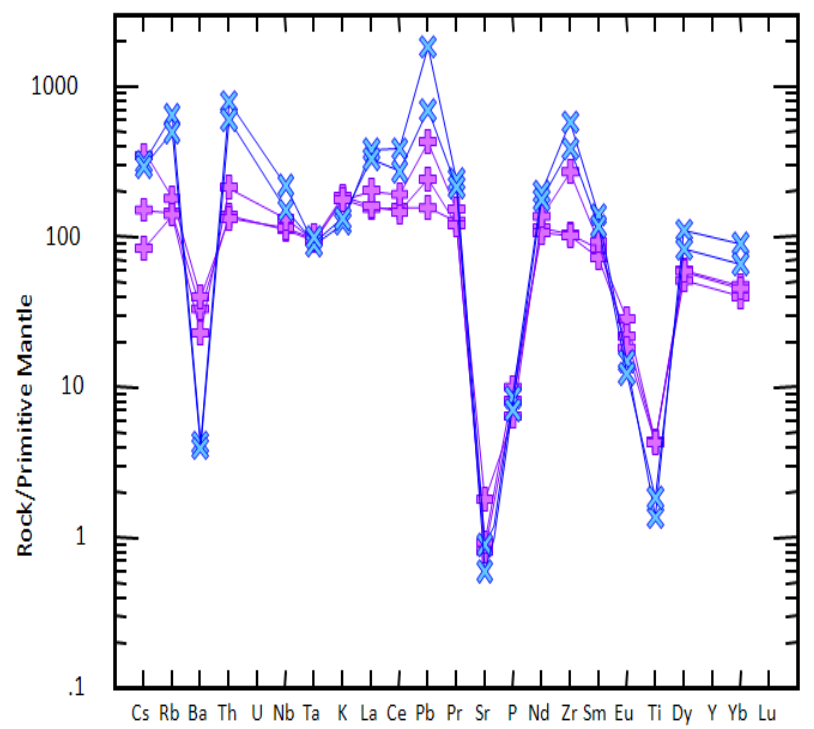

Figure 14. Primitive mantle-normalized multielement patterns for Dhiran granites. Normalization values are from Sun and McDonough,[53]

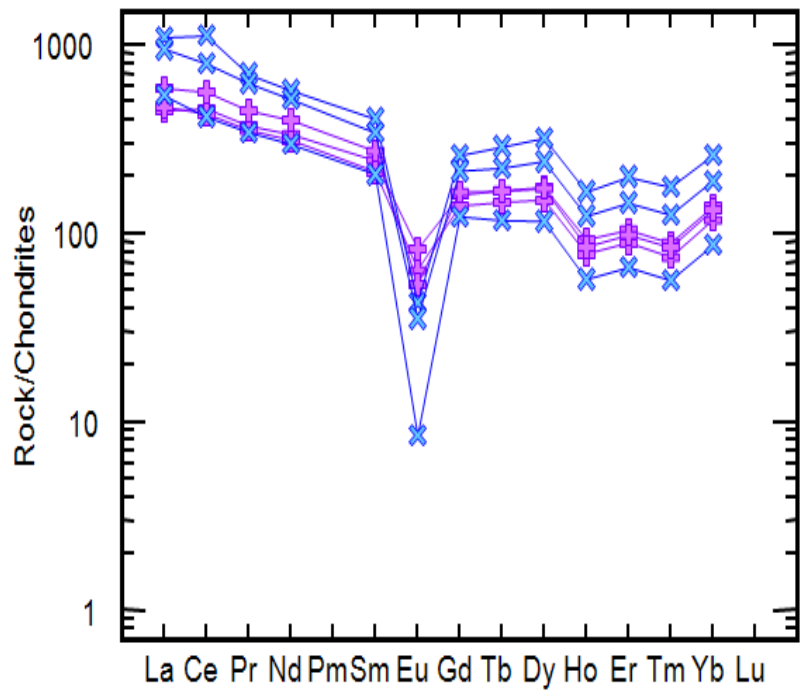

Figure 15. Condrite-normalized multielement patterns for Dhiran granites. Normalization values are from Sun and McDonough[53]

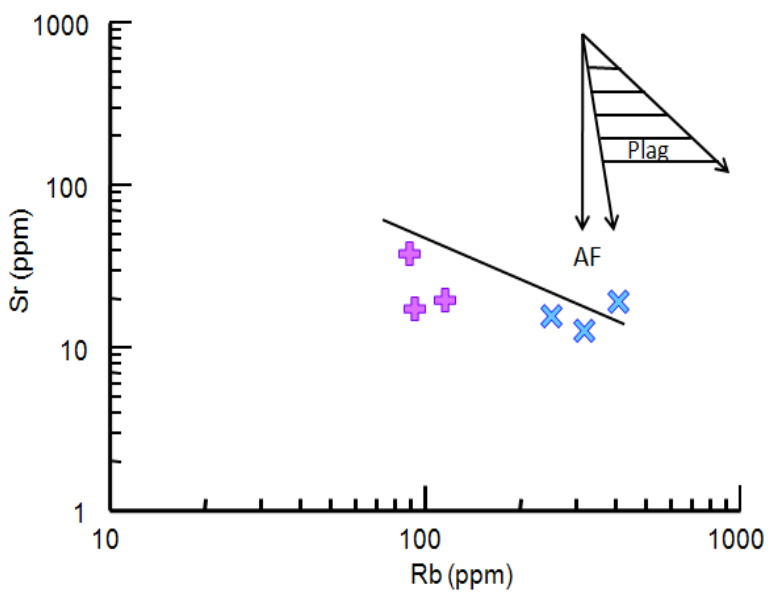

Figure 16. $\mathrm{Rb}$ vs $\mathrm{Sr}$ diagram. The fractional evolution of the plagioclase and alkali feldspar is after Arth,[54]

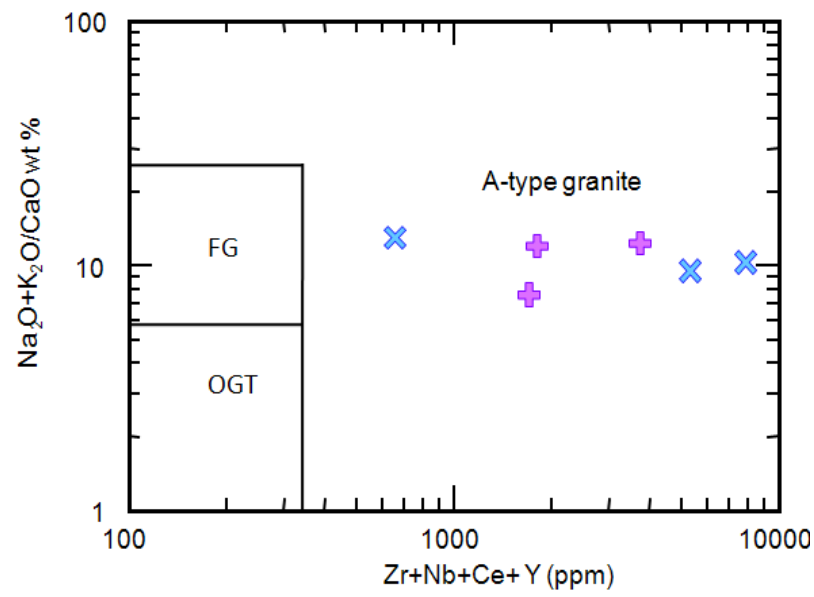

Figure 17. $\mathrm{Zr}+\mathrm{Nb}+\mathrm{Ce}+\mathrm{Y}$ vs $\mathrm{Na}_{2} \mathrm{O}+\mathrm{K}_{2} \mathrm{O} / \mathrm{CaO}$ diagram[2]. $\mathrm{FG}=$ Field for fractionated I-type granitoids. OGT= Field for I, $\mathrm{S}$ and $\mathrm{M}$ - type granitoids

The trace element aboundances of granites are comparable to those of typical anorogenic granite. They are characterized by high concentration of $\mathrm{Rb}, \mathrm{Nb}, \mathrm{Zr}, \mathrm{Ga}, \mathrm{Y}, \mathrm{Hf}, \mathrm{Th}, \mathrm{U}$ and prominent $\mathrm{Eu}$ anomalies with moderate LREE enrichment and flat HREE patterns (Fig. 14). Spidergrams show similar characteristics to those of pink granite and leucocratic granite with negative $\mathrm{Ba}, \mathrm{Sr}, \mathrm{P}$ and Ti anomalies, indicating either the retention of plagioclase and accessory minerals in the source during partial melting or their separation during fractionation (Fig. 15). However, these rocks show high $\mathrm{Zr}$ contents and have poor zircon yield, reflecting its high solubility in the alkaline magma ([44,45]). In the diagram Rb vs $\mathrm{Sr}$ (Fig. 16) and diagram indicates feldspar fractionation crystallization with minor plagioclase during the evolution of these rocks. The feldspar fractionated nature of the granitoids is indicated by the low $\mathrm{Sr}$ content and the negative $\mathrm{Eu}$ anomaly, suggesting plagioclase fractionation plays an important role in the evolution of A-type magmatism[46]. The granites are high in $\mathrm{SiO}_{2}, \mathrm{Fe}_{2} \mathrm{O}_{3}(\mathrm{t}), \mathrm{Na}_{2} \mathrm{O}+\mathrm{K}_{2} \mathrm{O}, \mathrm{Zr}, \mathrm{Hf}$, $\mathrm{Th}, \mathrm{U}, \mathrm{Nb}, \mathrm{Ta}, \mathrm{Y}, \mathrm{REE}, \mathrm{HPU}, \mathrm{Rb} / \mathrm{Sr}, \mathrm{Zr} / \mathrm{Rb}$ values and low in $\mathrm{CaO}, \mathrm{MgO}, \mathrm{Ba}, \mathrm{Sr}, \mathrm{Sc}, \mathrm{Ba} / \mathrm{Rb}, \mathrm{TiO}_{2} / \mathrm{Ta}$ values, suggesting their crustal origin ([19,20,47-50]). It also supported by their high $\mathrm{Nb}, \mathrm{Zr}$, Y and low Ti contents, characteristic of acid 
magmas generated within-plate tectonic environment[51].

Enrichment in the high field strength (HFS) elements is a characteristic feature of alkaline A-type granites in general. The high enrichment of these elements in the investigated granites confirm their A-type identity and exclude them from other granite type on the $(\mathrm{Zr}+\mathrm{Nb}+\mathrm{Ce}+\mathrm{Y})$ vs $\left(\mathrm{Na}_{2} \mathrm{O}+\mathrm{K}_{2} \mathrm{O}\right) / \mathrm{CaO}$ diagram (Fig. 17). All the granites samples lay away from $\mathrm{Sr}$ and arrange parallel to the $\mathrm{Ba}-\mathrm{Rb}$ side in the Ba-Rb-Sr ternary diagram (Fig.18). The relation between $\mathrm{Ba}, \mathrm{Sr}$ and $\mathrm{Rb}$ could be used in tracing differentiation trends in acid suites. Based on the distribution fields and evolution paths of El Bouseily and El Sokkary,[55], it is evident that all the studies samples plots in the strongly differentiated granites and normal granites field.

\section{Mineralization and Radioactive Element Distribution}

Presence of long lived isotopes of radioactive elements (U, $\mathrm{Th}, \mathrm{K})$ causes heat generation in the crust and their content are significant in understanding the nature of source magma and its mineralization. The radiogenic heat thus produced in the surface rocks play a significant role in the continental heat flow. Mineralization associated with A-type granite includes $\mathrm{Sn}, \mathrm{Mo}, \mathrm{Ba}, \mathrm{Nb}, \mathrm{W}, \mathrm{Ta}, \mathrm{F}, \mathrm{Be}, \mathrm{Li}$, and REE ([1],[56]). The largest deposits of Nb, Ta, Zr, Hf, Sr, Al, P, REE etc. are associated with alkaline magmatism [57]. Biste,[58] described the mineralized granites as containing relatively higher $\mathrm{Rb} / \mathrm{Sr}$, Li x $10^{-3} / \mathrm{K}$ and $\mathrm{Ba} / \mathrm{Sr}$ ratios and $\mathrm{Zr}$ and lower values of $\mathrm{K} / \mathrm{Rb}, \mathrm{Ba} / \mathrm{Rb}, \mathrm{Mg} / \mathrm{Li}$ and $\mathrm{Zr} / \mathrm{Sn}$. The granites of Dhiran area of MIS are enriched in $\mathrm{Rb}, \mathrm{Nb}, \mathrm{Y}, \mathrm{Zr}$, $\mathrm{Ta}, \mathrm{U}, \mathrm{Th}, \mathrm{Hf}, \mathrm{Pb}$ and REE and depleted in $\mathrm{Sr}, \mathrm{Ba}$ and Ti. The enrichment of $\mathrm{Zr}, \mathrm{Nb}$ and high $\mathrm{Zr} / \mathrm{Rb}$ ratio in the rocks indicate $\mathrm{Nb}-\mathrm{Sn}-\mathrm{W}$ mineralization ([14],[59]). High $\mathrm{Rb} / \mathrm{Sr}$ ratio and low $\mathrm{Ba} / \mathrm{Rb}$ ratio in granitoids indicate post magmatic and $\mathrm{Sn}-\mathrm{Nb}$ mineralization[60]. Leucocratic granite have more concentration of $\mathrm{Y}(164.13-542.55 \mathrm{ppm}), \quad \mathrm{Zr}$ (312.67-6568.28ppm), $\quad \mathrm{Nb} \quad(29.77-156.26 \mathrm{ppm}), \quad \mathrm{Hf}$ (11.57-79.68ppm), Pb (34.16-131.22ppm), Th (31.2967.62ppm), U (3.47-13.83), La (128.43-261.70ppm), Ce (147.26-690.73ppm), and Nd (139.74-270.02ppm) than Pink granites: Y (217.85-259.23ppm), Zr (1138.81- 3064.34ppm), $\mathrm{Nb}$ (80.47-95.52ppm), Hf (31.34-35.93ppm), $\mathrm{Pb}$ (11.30-30.65ppm), Th (11.93-18.12ppm), U (3.164.75ppm), La (106.60-140.09ppm), Ce (262.63-344.41ppm) and $\mathrm{Nd}$ (145.12-186.34ppm). High contents of $\mathrm{Ba}, \mathrm{Pb}, \mathrm{Th}, \mathrm{U}$, LREE and $\mathrm{Zr}$ in the leucocratic granite are possibly due to its notable contents of radioactive minerals such as Zircon, apatite and monazite. The concentration of $\mathrm{Y}$ in leucocratic granites (164.13-542.55ppm) and pink granites $(217.85-259.23 \mathrm{ppm})$ is much higher than the quoted average value of $40 \mathrm{ppm}$ for normal granites[61]. Anomalous $\mathrm{Zr}$ content is found in both the granites, 312.67-6568.28ppm for leucocratic granites and 1138.81-3064.34ppm, for pink granites. The $\mathrm{Zr}$ enrichment trend is accompanied by en- hance $\mathrm{U}$ and $\mathrm{Nd}$ associated with Nd-Sn-W mineralization[62-64] have reported variable amount of $\mathrm{Zr}$ (20 and $300 \mathrm{ppm})$ in the Sr-W-Mo-Cu related granitoids of the Australia and range granitoids of Malaysia respectively. Ga concentration is more in the pink granites $(40.14-57.60 \mathrm{ppm})$ than leucocratic granites (22.39-39.86ppm). The concentration of $\mathrm{Pb}$ in both the granites (34.16-131.22ppm and $11.30-30.65 \mathrm{ppm}$ ) are much higher than the quoted average abundance of $20 \mathrm{ppm} \mathrm{Pb}$ for normal granites[61]. Sn- bearing or productive granitoids contains $\mathrm{Pb}>15 \mathrm{ppm}$ whereas the Sn-barren or non productive granitoids has $\mathrm{Pb}<5 \mathrm{ppm}$. A-type anorogenic granites that are emplaced along rift zones or major lineaments characterized by high contents of $\mathrm{Ga}, \mathrm{Y}, \mathrm{REE}$ and $\mathrm{Nb}$, with notable depletion of $\mathrm{Ba}, \mathrm{Ti}, \mathrm{P}[65]$.

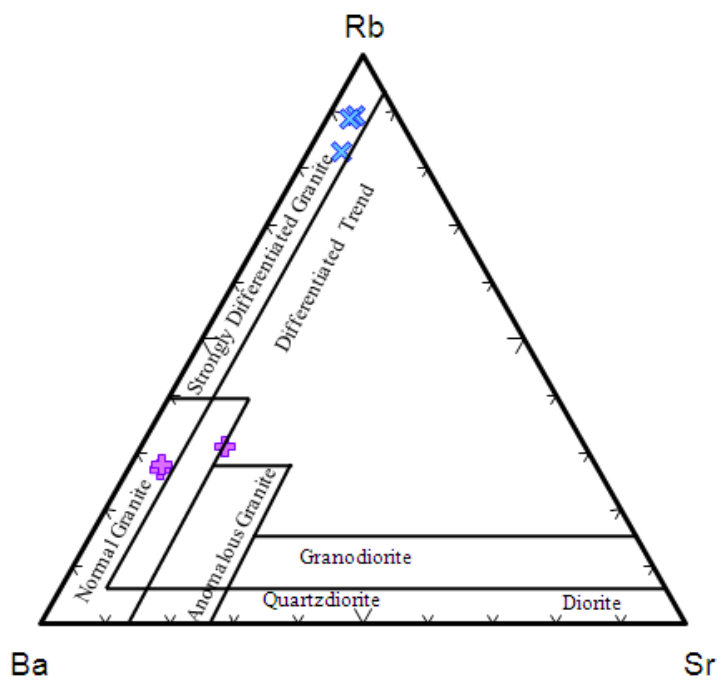

Figure 18. Ba-Rb-Sr ternary diagram for studied granites. Compositional fields and differentiation trends are after El Bouseily and El Sokkary,[55]

In these Granites viz. leucocratic granite generate higher value of $\mathrm{Ur}=32.59-53.76 ; \mathrm{HP}=4.84-8.75 \mu \mathrm{Wm}^{-3} ; \mathrm{HGU}=$ 11.52-20.84 than pink granite $(\mathrm{Ur}=18.19-22.69$; $\mathrm{HP}=$ 2.09-2.976 $\mu \mathrm{Wm}^{-3}$; HGU $\left.=4.98-7.07\right)$. The average concentration of $U$ and Th of the granites of the Dhiran area significantly higher (upto 3 times than the world concentration of $4 \mathrm{ppm}$ for $\mathrm{U}$ and $18 \mathrm{ppm}$ for Th in granite[66] and high upto 8 times than upper continental crust[67].

The concentration of $U$ and $T h$ of the both granites are shown in the Th-U diagram (Fig. 19). It exhibits positive correlation and both shows greater mobility. The distribution of $U$ and Th are much higher in leucocratic granite than Pink granite (Fig. 19). Granites in the upper parts of the continental crust usually have 3-4 ppm of $U$ and $10-15 \mathrm{ppm}$ of Th with a $\mathrm{Th} / \mathrm{U}$ ratio of 3.7[68]. The granites of the Dhiran area of MIS shows higher concentration of $U$ and $T h$ as compared to A-type granite \& rhyolite of Northwestern Ontario[69] and A-type rhyolite of St. Francois Mountains, Missouri[70] (Fig. 19). The enrichment of $U$ in granites appears to be due to fractional differentiation and that is indicates by the increase of $\mathrm{K}$ content of these granites. The $\mathrm{Th} / \mathrm{U}$ ratios of the granites are comparable and are fairly close to the upper crustal estimate of 3.8[67] (Fig. 20). The most Th and U 
enriched rocks sample have lower ratio of $\mathrm{Th} / \mathrm{U}$. Low $\mathrm{Th} / \mathrm{U}$ ratios in few sample may suggest fractionation of phase like Th-orthosilicate and Zircon[71]. In the K-U-Th diagram

(Fig. 21) granites plot near the Th-apex, indicating high content of Th (11.38-67.62ppm) in the samples, and hence the heat generation of Th (2.03-11.52 HGU) is much higher than U (1.97-8.61 HGU) and K (0.70-1.07 HGU). The average total heat generation value of $16.51 \mathrm{HGU}$ for leucocratic granites, 5.79 HGU for pink granites is much higher than average value of $3.8 \mathrm{HGU}$ for continental crust[72] and the value of 8.3 HGU obtained for the Peninsular India[73]. Since the heat generation value of $7 \mathrm{HGU}$ is taken as the boundary between the 'hot crust' and 'cold crust' the Dhiran granites (Leucocratic granite) data ( $>7 \mathrm{HGU}$ ) support that granites belongs to the 'hot crust' category. Both the granites show comparable heat production and heat generation value. The HP-Ur diagram (Fig. 22), shows good correlation of HP and Ur concentrations. The fields of the rhyolite of Jhunjhunu, MIS[74] and rhyolite of Mokalsar, MIS[14] is shown for comparison. The present studies samples are well comparable with Mokalsar Rhyolite.

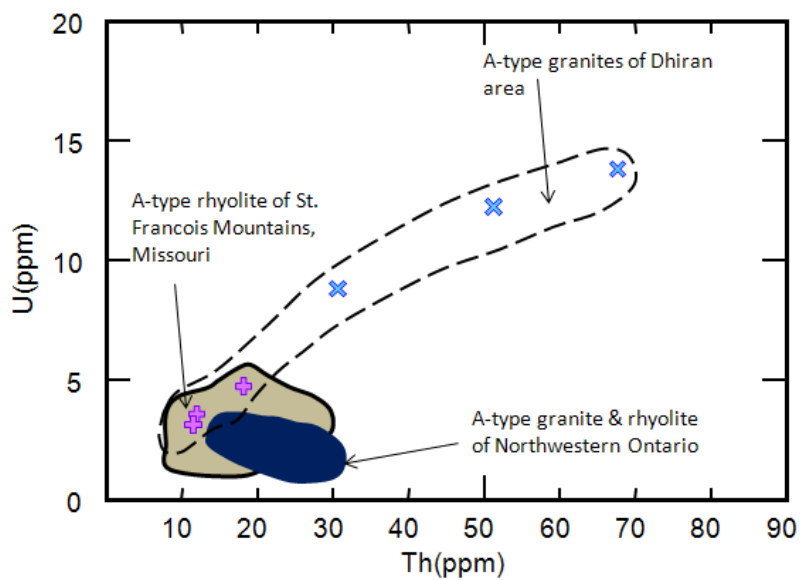

Figure 19. Plots of Th vs U. Fields of A-type rhyolite of St. Francois Mountains, Missouri[70] and A-type granite and rhyolite of Northwestern Ontario[69] are shown for comparison

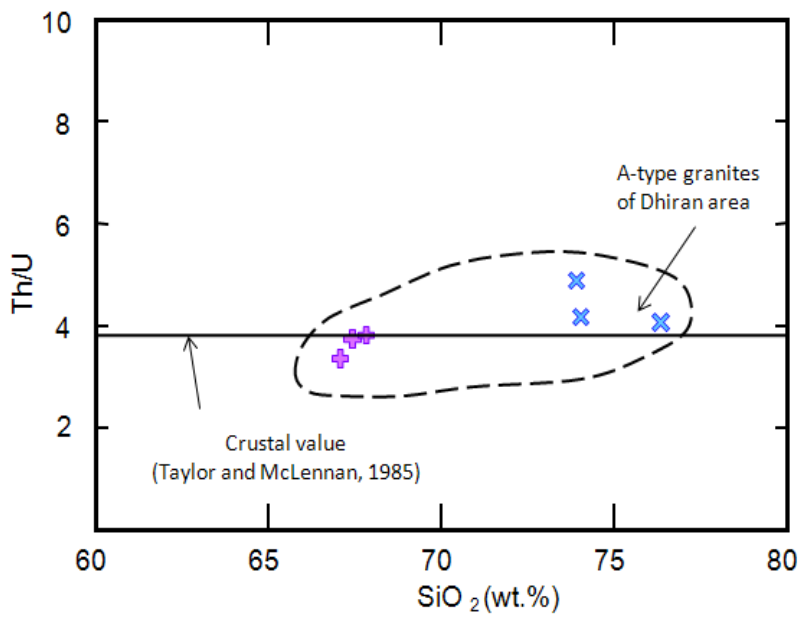

Figure 20. Plots $\mathrm{SiO}_{2}$ vs $\mathrm{Th} / \mathrm{U}$. Showing the boundary of Crustal value[67]

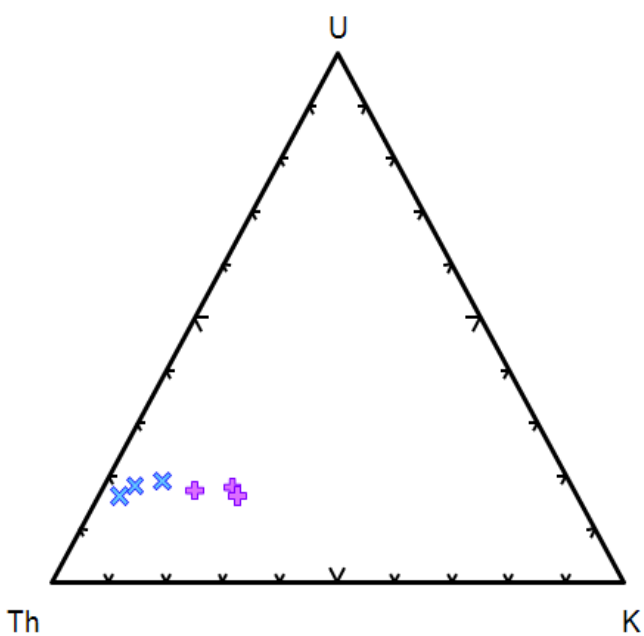

Figure 21. Plots of K-U-Th ternary diagram showing the concentration of $\mathrm{K}, \mathrm{U}$ and $\mathrm{Th}$ of the Dhiran granites

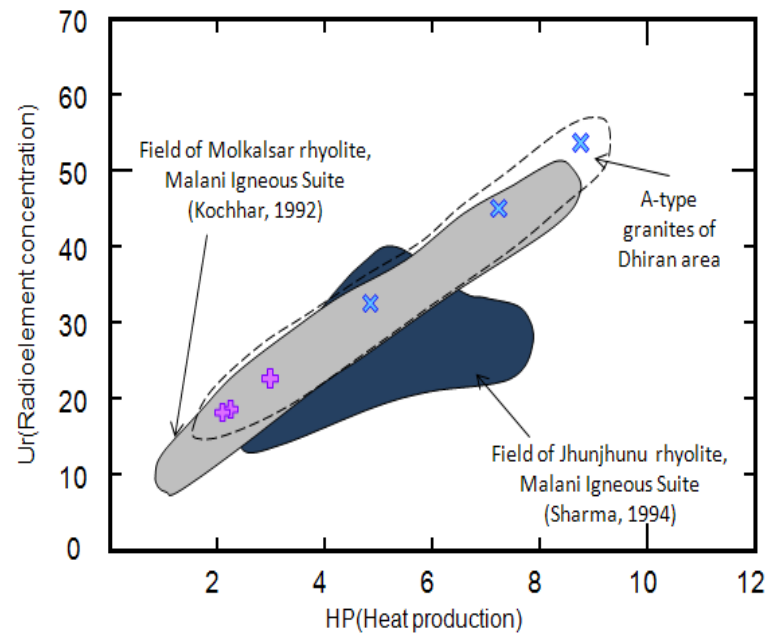

Figure 22. HP vs Ur diagram showing comparison of Jhunjhunu rhyolite, Malani Igneous Suite[74] and Molkalsar rhyolite Malani igneous Suites[14]

\section{Petrogenesis}

A number of petrogenetic models have been proposed for the origin of A-type granites, including high fractional crystallization of mantle-derived mafic magmas, with or without crustal assimilation ([75],[76]) and partial melting of pre-existing crustal materials ([4],[77]). Possibly, A-type magma does not represent a particular geologic setting, but rather similar end-products derived through different processes ([8],[78]). The obtained geochemical data indicates that the partial melting hypothesis is more applicable for the Dhiran granites according to the following reasons: The presence of a compositional gap in the major and trace elements, as well as the irregularity in REE distributions among the studied granites indicate a different magma source. The fractionation of basaltic melts, which is dominated by plagioclase, pyroxene and olivine in the lower to middle crust, would produce magmas with very large negative europium anomalies[79]. Both the granites show very large negative europium anomalies. Eby,[8] divided the A-type granitoids 
into A1 and A2 chemical groups, based on tectonic affiliation $(\mathrm{A} 1=$ truly anorogenic rifting, $\mathrm{A} 2=$ postcolisional $)$ and the $\mathrm{Y} / \mathrm{Nb}$ ratios to differentiate between mantle $(\mathrm{Y} / \mathrm{Nb}<1.2)$ and crustal $(\mathrm{Y} / \mathrm{Nb}>1.2)$ origin. A-type granitoids have $\mathrm{Y} / \mathrm{Nb}$ ratios (2.4-5.5) greater than 1.2 and they plot clearly in the $\mathrm{A} 2$ granite field in the $\mathrm{Y} / \mathrm{Nb}-\mathrm{Rb} / \mathrm{Nb}$ binary diagram (Fig. 23). Accordingly, these chemically defined A-type granites correspond to magmas derived from a crustal source. In the $\mathrm{Y}-\mathrm{Nb}$ variation diagram (Fig. 24), the $\mathrm{Y}$ and $\mathrm{Nb}$ increase systematically along well defined positive trends as shown by the distinct lineup of data points (Fig. 24) also, Y is typically concentrated in accessory minerals, commonly zircon, as $\mathrm{Y}$ replaces $\mathrm{Zr}[80]$. As expected, a negative correlation between $\mathrm{Ba}$ and $\mathrm{Rb}$ is observed in Fig. 25, which shows a gradual decrease in Ba with increasing $\mathrm{Rb}$. This is possibly the result of fractional crystallization of the alkali feldspar (perthite). It should be noted that these granitoid indicates control by crystal-melt equilibrium[81] and the magmatic processes control the evolution of this granitoid, were crystal-liquid equilibrium processes with minimal involvement of post-magmatic aqueous fluid phase interactions. Dhiran granites produce a well-defined negative trend showing gradual decrease in the $\mathrm{Rb} / \mathrm{Sr}$ ratios linearly with increasing concentration of Sr. It should be noted that the depletion in $\mathrm{Sr}$ and $\mathrm{Ba}$, which is typically associated with enrichment in $\mathrm{Rb}$ in alkaline felsic melts (Fig. 26) is interpreted to have been caused by internal differentiation of the parent granitic magma. Sr and Ba are typically removed with the fractionation of the feldspars, whereas $\mathrm{Rb}$ (a highly incompatible element) is gradually being enriched in late stage residual melts. Spider diagrams (Fig. 14) have pronounced $\mathrm{Ba}, \mathrm{Sr}$, and $\mathrm{Ti}$ troughs and ales obvious $\mathrm{Nb}$ trough. The $\mathrm{Ba}$, $\mathrm{Sr}$, and $\mathrm{Ti}$ depletion could be related to fractionation of plagioclase and ilmenite. The $\mathrm{Nb}$ trough is a strong indicator of continental crust involvement in magma processes ([82],[83]), as they are very characteristic feature of rocks formed of granites derived from a crustal source. The deep negative Ti anomaly or depletion may be linked with crustal contamination phenomena and/or involvement of continental materials ([84],[85]). However, the strong depletion and very low abundances of $\mathrm{Ba}, \mathrm{Sr}, \mathrm{Eu}$ and $\mathrm{Ti}$, which sometime drop below those of primitive mantle, require an advanced fractionation of feldspars and ilmenite or titanomagnetite in upper crustal conditions. All of the REE patterns have strong negative Eu anomalies and exhibit concave upward shapes of obvious negative slopes due to light REE enrichment relative to middle and heavy REE. The heavy REE are not greatly depleted, but in turn are comparable or even sometimes slightly enriched relative to middle REE, suggesting absence of garnet in the source, since heavy REE are highly compatible in garnet[82]. This further indicate that, if mantle participation is assumed in the source material, a shallow mantle is preferred rather than deep one where spinal stability is favored rather than garnet[86]. The fraction of the heavy rare earth element (HREE) is considerable pronounced with significantly high $(\mathrm{Gd} / \mathrm{Yb})_{\mathrm{N}}$ ratios ranging between 1.25 and 1.78 , suggesting that more HREE-bearing minerals, such as hornblende and pyroxene, might have been fractionated during the formation of these granites. The similarities between Arabian-Nubian Shield (ANS) and Malani supercontinent in respect of age, ring structure, tectonic environment and element compositions which provide a new approach for the search of economic mineralization[87].

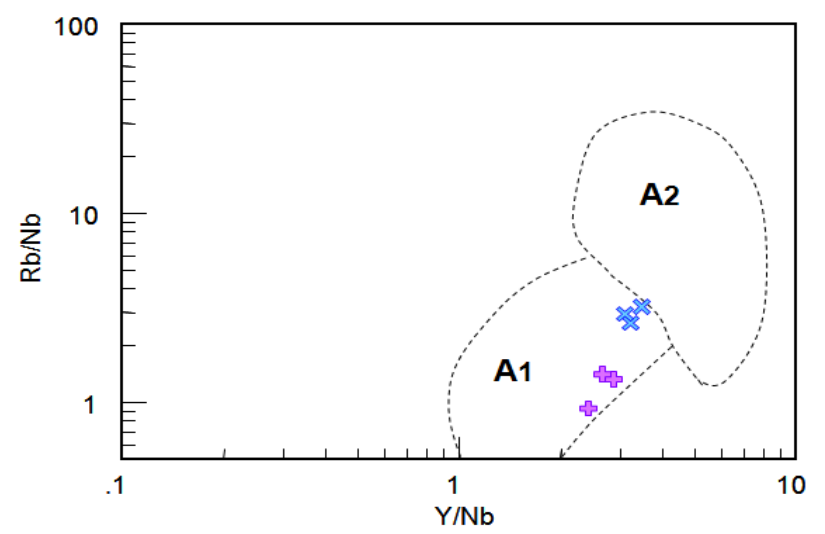

Figure 23. Plots of the studies A-type granites on $\mathrm{Rb} / \mathrm{Nb}$ vs. $\mathrm{Y} / \mathrm{Nb}$ binary diagram[8] A1 and A2 granitoids (A1=Truly anorogenic rifting, A2= Postcollisional)

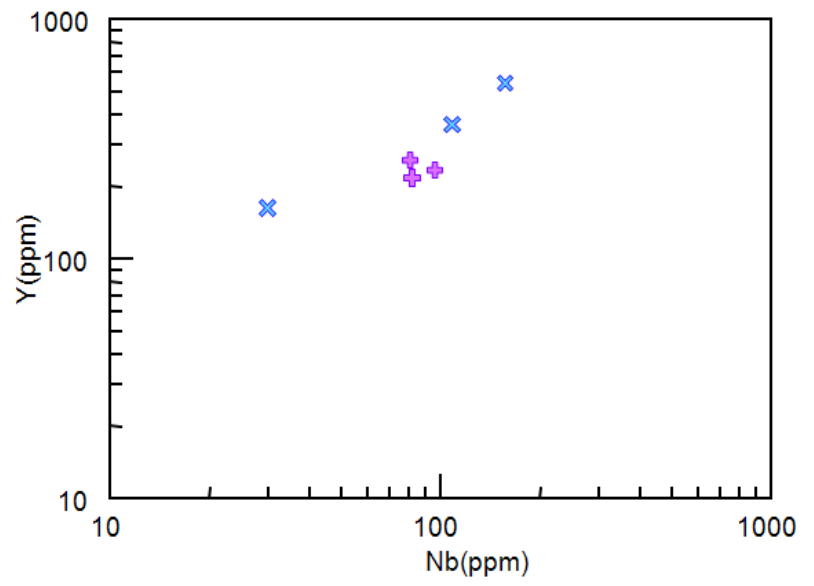

Figure 24. $\mathrm{Nb}$ vs $\mathrm{Y}$ variation diagram showing a well-defined trend, which displays the systematic increase in both elements

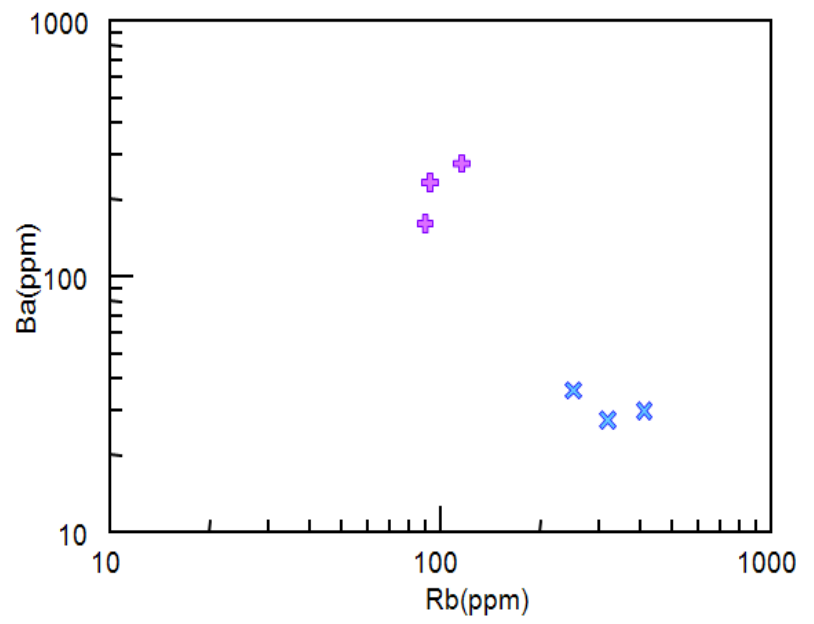

Figure 25. $\mathrm{Rb}$ vs $\mathrm{Ba}$ variation diagram showing a well-defined trend, which displays the systematic increase of $\mathrm{Rb}$ with decrease of $\mathrm{Ba}$ 


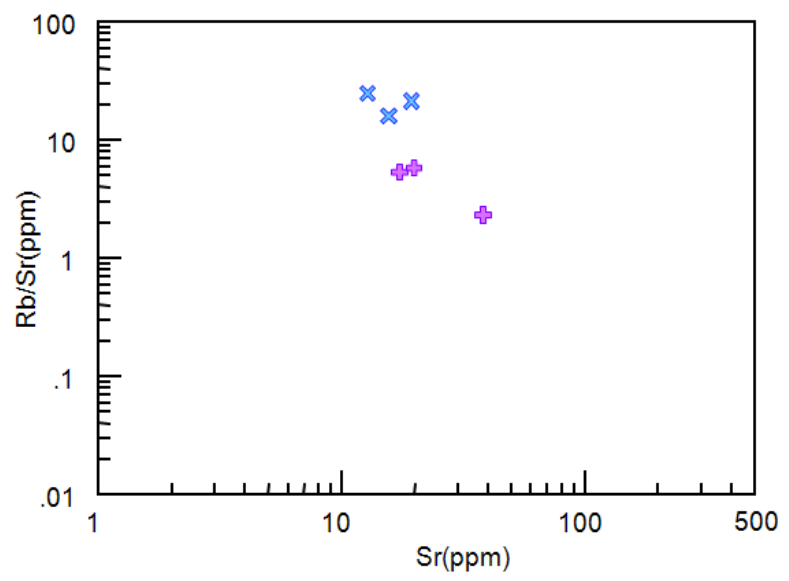

Figure 26. $\mathrm{Rb} / \mathrm{Sr}$ vs $\mathrm{Sr}$ diagram showing that the $\mathrm{Rb} / \mathrm{Sr}$ ratios increase with decreasing $\mathrm{Sr}$ contents

According to Bonin,[88], A-type magma move up and pass through the crust. Their levels of emplacement from the mantle-crust boundary to the surface are controlled mainly by the mechanical discontinuities with solid rocks, favoring propagation of buoyant magmas and by wall rock density and strength, tending to arrest them, and then crust operates as a density filter for migrating magmas.

\section{Conclusions}

Anorogenic A-type granitoids of the Dhiran area of MIS, Western Peninsular India are classified chemically as ferroan, alkaline, peralkaline and peraluminous, A-type granites. This A-type granites are highly evolved in composition (65.85-77.74 $\mathrm{wt} \% \mathrm{SiO}_{2}$ and $\mathrm{DI}$ values of 83.36-92.45) and display the typical geochemical characteristics of A-type granites with high $\mathrm{SiO}_{2}, \mathrm{Na}_{2} \mathrm{O}+\mathrm{K}_{2} \mathrm{O}, \mathrm{FeO} * \mathrm{MgO}, \mathrm{Ga} / \mathrm{Al}, \mathrm{Zr}$, $\mathrm{Nb}, \mathrm{Ga}, \mathrm{Y}, \mathrm{Ce}$, and REE and low $\mathrm{CaO}, \mathrm{MgO}, \mathrm{Ba}, \mathrm{Sr}$, they exhibit significantly high $\mathrm{Rb} / \mathrm{Sr}$ ratios and negative Eu anomalies. Their trace and REE characteristics indicate predominant formation by partial melting of continental crust. Although these A-type granites display almost all the conventional typical characteristic of anorogenic within plate magmas, comprehensive integrated investigations revealed that they are corresponding to magmas derived from a crustal source that has gone through a hot-spot tectonics. The extremely high $\mathrm{Rb} / \mathrm{Sr}$ ratios combined with the obvious $\mathrm{Sr}, \mathrm{Ba}, \mathrm{P}, \mathrm{Ti}$, and Eu depletions clearly indicates that these A-type granites were highly evolved and require advanced fractional crystallization in upper crustal conditions and argue against its formation by fractional crystallization of a parental mafic magma.

High to medium content of radioactive element (U, Th, K) heat generation of anorogenic, A-type granites of Dhiran Area, MIS, has been reported. These granites are classified as high to medium Heat Production (HP). Leucocratic granites and pink granites of the Dhiran area shows higher average value of total Heat Generation Unit than the average value of $3.8 \mathrm{HGU}$ for the continental crust. The high Heat Generation Unit values of the Dhiran granite indicates 'hot crust' category and a possible linear relationship between the surface heat flow and crustal heat generation in the MIS. Since, the HHP acidic rocks are associated with rare metal mineralization and based on the characteristic of $\mathrm{U}, \mathrm{Th}, \mathrm{Nb}$, $\mathrm{Zr}, \mathrm{Zn}, \mathrm{Pb}$ and $\mathrm{REE}$, it is suggested that the granitoids of Dhiran of MIS have the potential for rare metal and rare earth elements mineralization.

Dhiran A-type magma move up and pass through the continental crust. Their levels of emplacement from the mantle-crust boundary to the surface are controlled mainly by the mechanical discontinuities with solid rocks, favoring propagation of buoyant magmas and by wall rock density and strength, tending to arrest them, then crust operates as a density filter for migrating magmas. Hence, the magmatism of the granites of Dhiran area, MIS are related to intraplate, crust mantle interaction, anorogenic, within plate, A-type, subvolcanic setting and emplaced in the extensional tectonic regime.

\section{ACKNOWLEDGEMENTS}

The first author is highly indebted to Dr. V. Venkateswarlu, Director (additional charge), National Institute of Rock Mechanics for permission to publish the paper and he also thankful to Dr. A.K. Naithani \& Dr. G.R Adhikari, National Institute of Rock Mechanic for their significant help. Authors are thankful to Dr. V. Balaram National Geophysical Research Institute, Hyderabad and Prof. V. Rajamani, SES, Jawahalal Nehru University, New Delhi for support and encouragement during chemical analyses.

\section{REFERENCES}

[1] Collins, W. J., Beams, S.D., White, A.J.R., Chappell, B.W., "Nature and origin of A-type granites with particular reference to Southeastern Australia", Contributions to Mineralogy and Petrology, vol. 80, pp. 189-200, 1982.

[2] Whalen, J. B., Currie, K. L., Chappell, B. W., “A - type granites: Geochemical characteristics, discrimination and petrogenesis", Contributions to Mineralogy and Petrology, vol. 96, pp. 407-419, 1987.

[3] Eby, G.N., "The A-type granitoids: A review of their occurrence and chemical characteristics and speculations on their petrogenesis", Lithos, vol. 26, pp. 115-134, 1990.

[4] Landenberger B, Collins W. J., "Derivation of A-type granites from dehydrated charnockitic lower crust: evidence from the Chaelundi complex Eastern Australia", Journal of Petrology, vol. 37, pp. 145-170, 1996.

[5] Clemens, J. D., Holloway, J.R. and White, A.J.R., "Origin of the A-type granite: experimental constraints", American Mineralogist, vol. 71, pp. 317-324, 1986.

[6] Anderson, J.L., "Proterozoic anorogenic granite plutonism of North America", Geological Society of America, vol. 161, pp. 133-154, 1983. 
[7] Creaser, R. A., Price, R.C and Wormald, R.J., "A-type granites revisited: Assessment of a residual source model", Geology, vol. 19, pp. 163-166, 1991.

[8] Eby, G.N., "Chemical subdivision of A-type granitoids: petrogenesis and tectonic implications", Geology, vol. 20, pp. 641-644, 1992.

[9] Beyth M, Stern R.J, Altherr R, Kröner A., "The Late Precambrian Timna igneous complex, southern Israel: evidence for comagmatic- type sanukitoid monzodiorite and alkali granite magma”, Lithos, vol. 31, pp. 103-124, 1994.

[10] Skjerlie K.P and Johnston A.D., "Vapor-absent melting at 10 kbar of a biotite- and amphibole-bearing tonalitic gneiss: implications for the generation of A-type granites", Geology, vol. 20, pp. 263-266, 1992.

[11] King, P.L., White, A.J.R., Chappell, B.W., Allen, C.M., "Characterization and origin of aluminous A-type granites from the Lachlan Fold Belt, Southeastern Australia", Journal of Petrology, vol. 38, pp. 371-391, 1997.

[12] Skjerlie K.P and Johnston A.D., "Fluid-absent melting behavior of an F-rich tonalitic gneiss at mid-crustal pressure: implication for the generation of anorogenic granites" Journal of Petrology, vol. 34, pp. 785-815, 1993.

[13] Martin, R. F., “A-type granites of crustal origin ultimately result from open-system fenitization-type reactions in an estensional environment", Lithos, vol. 91, pp. 125-136, 2006.

[14] Kochhar, N., "Mineralization associated with A - type Malani magmatism, Northwestern peninsular India", In: Sarkar, S. C. (Ed.), Metallogeny related to tectonic of the Proterozoic mobile belts. Oxford-IBH, New Delhi, pp. 209-224, 1992.

[15] Baskar, R., Sharma, S., "An assessment of the rare metal potential of the granitoids of Siwana, Jalor, Jhunjhunu and Tusham, North Western Peninsular India", Current Science, vol. 66, no. 1, pp. 67-69, 1994.

[16] Jain, R .B., Miglani, T. S., Kumar, S., Swarnkar, B. M., Singh, R., "Rare metal and rare earth rich peralkaline, agpaiitic granitoid dykes of Siwana Ring Complex, district Barmer, Rajasthan”, Current Science, vol. 70, pp. 854-858, 1996.

[17] Vallinayagam, G., "Nb, Zr, REE rich acid dyke rocks from the Piplun area, Siwana Ring Complex, Western Rajasthan, India”, In: Paliwal, B.S. (Ed.), Geological Evolution of Northwestern India. Scientific Publishers, Jodhpur, pp. 94-102, 1999.

[18] Vallinayagam, G., "A report on rare metals and rare earths in the Siwana Ring Complex, Rajasthan”, Journal of Applied Geochemistry, vol. 2, pp. 387-391, 2004.

[19] Singh, A. K., Singh, R. K. B., Vallinayagam, G., “Anorogenic acid volcanic rocks in the Kundal area of the Malani Igneous Suite, Northwestern India: Geochemical and petrogenetic studies", Journal of Asian Earth Sciences, vol. 27, pp. 544-557, 2006.

[20] Singh, A. K., Vallinayagam, G., "Radioactive element distribution and rare-metal mineralization in anorogenic acid volcano-plutonic rocks of the Neoproterozoic Malani Felsic Province, Western Peninsular India", Journal of Geological Society of India, vol. 73, pp. 837-853, 2009.

[21] Chaudhary, A. K., Gopalan, K., Anjaneya Sastry, C., "Present status of the geochronology of the Precambrian rocks of Ra- jasthan", Tectonophysics, vol. 105, pp. 131-140, 1984.

[22] Bhushan,S.K., "Late Proterozoic continental growth: implecation from geochemistry of acid magmatic events of West Indian craton, Rajasthan", Memorior Geological Society of India, vol. 34, pp. 339-355, 1995.

[23] Bhushan, S.K., "Stratigraphic position of Marwar Supergroup, Western Rajasthan", In: Abstract volume of the National Seminar on Stratigraphic Boundary Problems in India. Jammu, pp. 5-6, 1988.

[24] Kochhar, N., "Malani Igneous Suite: Hot-spot magmatism and cratonization of the Northern part of the Indian shiled" Journal of Geological Society of India, vol. 25, pp. 155-161, 1984.

[25] Bhushan, S.K., Chittora, V.K., "Late Proterozoic bimodal assemblage of Siwana subsidence structure, Western Rajasthan, India", Journal of Geological Society of India, vol. 53, pp. 433-453, 1999.

[26] Taylor, H. P. J., Forester, R. W., "Low $\mathrm{O}_{18}$ igneous rocks from the intrusive complexes of Skye, Mull and Ardnamurchan, Western Scotland", Journal of Petrology, vol. 12, pp. 465-498, 1974.

[27] Kinnaird, J.A.,"Hydrothermal alteration and mineralization of the alkaline anorogenic ring complex of Nigeria", Journal of African Earth Science, vol. 3, pp. 229 - 251, 1985.

[28] Groover, D.I., Mac Carthy, T.S., "Fractional crystallization and origin of tin deposits in granitoids", Mineral Deposita, vol. 13, pp. 11-26, 1978.

[29] Parson, I., "Alkali feldspar and Fe-Tin oxide exsolution textures as indicators of the distribution and subsolidus effects of magmatic "water" in the Klokken layered syenite intrusion, South Greenland", Transactions Research Society, Edinburgh, Earth Science, vol. 71, pp. 1-12, 1980.

[30] Yund, R.A., Ackermand, D., "Development of perthite microstructures in the strom King granites, New York", Contributions to Mineralogy and Petrology, vol. 70, pp. 273-280, 1979.

[31] Streckeisen, A. L., "IUGS Subcommission on the systematics of Igneous Rocks: Classification and nomenclature of volcanic rocks, lamprophyres, carbonatites and melilitic rocks; recommendation and suggestions", Neues Jahrbuch für Mineralogie, Abhandlungen, vol. 134, pp. 1-14, 1978.

[32] Maniar, P.D., Piccoli, P.M., "Tectonic discrimination of granitoids", Geological Society of America Bulletin, vol. 101, pp. 635-643, 1989.

[33] Rickwood P.C., "Boundary lines within petrologic diagrams which use oxides of major and minor elements", Lithos, vol. 22, pp. 247-263, 1989.

[34] Rogers, J.J.W., Greenberg, J.K., "Trace element in continental margin magmatism: Pt. III Alkali granites and their relationship to the Cratonisation: summary", Bulletin of Geological Society of America, vol. 92, pp. 6-9, 1981.

[35] Vallinayagam, G., "Minerals chemistry of Siwana Ring complex, W. Rajasthan, India", The Indian Mineralogist, vol. 31, pp. 37-47, 1997.

[36] Baskar, R., Kochhar, N., "Titan-aegirine from the peralkaline Siwana granite, Western Rajasthan, India”, Journal of Ap- 
plied Geochemistry, vol. 2, pp. 133-136, 2006.

[37] Frost, B. R., Barnes, C. G., Colins, W.J., Arculus, R.J., Ellis, D.J., Frost C.D., "A geochemical classification for granitic rocks", Journal of Petrology, vol. 42, pp. 2033-2048, 2001.

[38] Petro, W.L.T, Vogel T.A, Willband J.T., "Major element chemistry of plutonic rocks suites from compressional and extentional plate boundaries", Chemical Geology, vol. 20, pp. 217-235, 1979.

[39] Abdel-Rahman A.M, El-Kibbi M.M., "Anorogenic magmatism: chemical evolution of the Mount El-Sibai A-type complex (Egypt), and implications for the origin of Within- Plate Felsic magmas", Geological Magazine, vol. 138, pp. 67-85, 2001.

[40] Pearce J.A, Harris N.B.W, Tindle A.G., "Trace element discrimination diagrams for the tectonic interpretation of granitic rocks", Journal of Petrology, vol. 25, pp. 959-983, 1984.

[41] Stern R.J and Gottfried D., "Discussion of the paper "Late Pan- African magmatism and crustal development in northeastern Egypt", Geological Journal, vol. 24, pp. 371-374, 1989.

[42] Batchelor, R.A., Bowden, P., "Petrogenetic interpretation of granitoid rock series using multicationic parameters", Chemical Geology, vol. 48, pp. 43-55, 1985.

[43] Eby, G.N., Kochhar, N., "Geochemistry and petrogenesis of the Malani Igneous Suite, North Peninsular India", Journal of Geological Society of India, vol. 36, pp. 109-130, 1990.

[44] Bowden, P., "Zirconium in younger granites of Northern Nigeria" Geochimica et Cosmochimica Acta, vol. 30, pp. 985-993, 1966.

[45] Kochhar, N., Sharma, R., Vallinayagam, G., "A note on tin-bearing potential of granites of the Jhunjhunu area, district Jhunjhunu, Rajasthan”, Indian Minerals, vol. 45, pp. 99-104, 1991.

[46] Stony, M., "Trachytic pyroclastic from Agua de volcano, Sao Miquel Azores: evolution of a magma body over 4000 years", Contribution to Mineralogy and Petrology, vol. 12, pp. 423-432, 1981.

[47] Vallinayagam, G., Kochhar, N., "Geochemical characterization and petrogenesis of A-type, granites and the associated acid vocanics of the Siwana Ring Complex, Northern Peninsular, India", In: Paliwal, B.S. (Ed.), The Indian Precambrian, Scientific Publishers, Jodhpur, pp. 460-48, 1998.

[48] Singh, A. K., and Vallinayagam, G., "Geochemistry of the A-type granite from the Kundal area of Malani Igneous Suite, District Barmer, Rajasthan: Implications for rare metal mineralization", Journal of Applied Geochemistry, vol. 5, pp. 16-25, 2003.

[49] Vallinayagam, G., "Occurrence of Zinc rich A-type granites in the Trans-Aravalli anorogenic ring complexes, Northwestern India”, In: Shrivastava, K.L. (Ed.), Economic Mineralization. Scientific Publishers, Jodhpur, pp. 205-209, 2009.

[50] Vallinayagam, G and Naresh Kumar, "Mineralization potential of the granites from Nakore ring complex, Barmer District, W. Rajasthan, India: A geochemical perspective", Journal of Applied Geochemistry, vol. 12, no. 3, pp. 375-387, 2010.
[51] Pearce, J.A., Norry, M.J., "Petrogenetic implications of Ti, Z, $\mathrm{Y}$ and $\mathrm{Nb}$ variations in volcanic rocks", Contributions to Mineralogy and Petrology, vol. 69, pp. 33-47, 1979.

[52] Pearce, J., "Sources and settings of granitic rocks", Episodes, vol. 19, pp. 120-125, 1996.

[53] Sun, S.S., McDonough, W.F., "Chemical and isotopic systematics of oceanic basalts: Implications for mantle composition and processes", In: Norry, M.J., Saunders, A.D. (Eds.), Magmatism in the ocean Basin. Special Publication, Geological Society, no. 42, pp. 313-345, 1989.

[54] Arth, J.G., "Behaviour of trace elements during magmatic process summary of theoretical models and their applications", Revised Geophysical Space Physics, vol. 15, pp. 96-104, 1976.

[55] El Bouseily A. M, El Sokkary A. A., "The relation between $\mathrm{Rb}, \mathrm{Ba}$ and $\mathrm{Sr}$ in granitic rocks", Chemical Geology, vol. 16, pp. 207-219, 1975.

[56] Pitcher, W.S., "Granite type and tectonic environment", In: Hsu, K. (Ed.), Mountain building process. Academic Press, London, pp. 19-40, 1983.

[57] Ovchinnikov, L.N., Deposits of Lithophile rare metals. Nedra, Moscow, 1980.

[58] Biste, M., "Die Anwendung geochemischer Indiakatoren auf die Zinn-Hoffigkeit herzynischer granite in sud-Sardinien", Berl. Geowiss. Abh. Vol. 18, no.1, 1979.

[59] Khin Zaw., "Geological, petrological and geochemical characteristics of granitoid rocks in Burma: With special reference to the associated W-Sn mineralization and their tectonic setting", Journal of Southeast Asian Earth Science, vol. 4, no. 4, pp. 293-335, 1990.

[60] Ekwere, S.J., "Li, $\mathrm{F}$ and $\mathrm{Rb}$ content and $\mathrm{Ba} / \mathrm{Rb}$ and $\mathrm{Rb} / \mathrm{Sr}$ ratios as indicators of postmagmatic alteration and mineralization in the granitic rocks of banks and Ririwqai younger complexes, Northern Nigerian", Mineral Deposita, vol. 20, pp. 89-93, 1985.

[61] Levinson, A.A., Introduction to Exploration Geochemistry. Applied Publishing Limited Wilmette, Illinois, 1974.

[62] Imeokparia, E.G., "Rare metal mineralization in granitic rocks of the Tangalo anorogenic complex, Northern Nigeria", Mineral Deposita, vol. 20, pp. 181-188, 1985.

[63] Flinter, B. H., Hesp, W. R., Rigby, D., "Selected geochemical mineralogical and petrological features of granitoids of the New England complex, Australia and their relation to Sn, W, Mo and $\mathrm{Cu}$ mineralization”, Economic Geology, vol. 67, pp. 1241-1262, 1972.

[64] Hutchison, C.S., "Granite emplacement and tectonic subdivision of Peninsular Malaysia", Bulletin of Geological Society of Malaysia, vol. 9, pp. 187-207, 1977.

[65] Dhana Raju, R., "Uranium exploration in the Proterozoic terrains of India: a petrometallographic study and implications on target selection", Exploration and Research for Atomic Minerals, vol. 8, pp. 173-186, 1995.

[66] Rogers, J. J. W., Adams, J. A. S, Thorium. In: Wedepohl, K.H. (Ed.), Handbook of Geochemistry. Springer-Verlag, Berlin, pp. 11-14, 1969.

[67] Taylor, S.R., McLennan, S.M., The Continental Crust: its 
composition and evolution. Black-well Scientific publications, Oxford, 1985.

[68] Wesserburg, G.G., "Relative contribution of $\mathrm{U}$, Th and $\mathrm{K}$ to heat production in the Earth's Crust", Science, vol. 143, pp. 465- 467, 1964.

[69] Hollings, P., Fralick, P., Kissin, S., "Geochemistry and geodynamic implications of the Mesoproterozoic English Bay granite-rhyolite complex, Northwestern Ontario", Canadian Journal of Earth Sciences, vol. 41, pp. 1329-1338, 2004.

[70] Menuge, J.F., Brewer, T.S., Seeger, C.M., "Petrogenesis of metaluminous A-type rhyolite from the St. Francois Mountains, Missouri and Mesoproterozoic evolution of the Southern Laurentian margin", Precambrian Research, vol. 113, pp. 269-291, 2002.

[71] Bea, F., "Residence of REE, Y, Th and U in granites and crustal protoliths implications for the chemistry of crustal melts", Journal of Petrology, vol. 37, pp. 521- 552, 1996.

[72] Taylor, S.R., "Trace element abundances and the chondritic earth model", Geochimica et Cosmochimica Acta, vol, 28, pp. 1989-1999, 1964

[73] Dhana Raju, R., Singh, J.P and Bhattacharya, T.K., "A preliminary stydy on radioactive heat generation of some Precambrian granitic rocks of India", Indian Journal of Earth Science, vol. 10, no. 2, pp. 112-119 1983.

[74] Sharma, R., "High heat production (HHP) granites of Jhunjhunu area, Rajasthan, India", Bulletin of Indian Geological Association, vol. 27, pp. 55-61, 1994.

[75] Volker R.A., Feigenson M.D., Pation L.C., Delaney J.S., and Drake A.A. Jr., "Sr and Nd isotopic composition, age and petrogenesis of a-type granitoids of the Vernon supersuite, New Jersey Highlands, 'USA", Lithos, vol. 50, pp. 325-347, 2000.

[76] Litvinovsky B.A, Jahn B M, Zanvilevich A.N, Saunders A, Poulain S., "Petrogenesis of syenite-granite suites from the Bryansky complex(Transbaikalia, Russia): implications for the origin of A-type granitoid magmas", Chemical Geology, vol. 189, pp. 105-133, 2002.

[77] Patino Douce A. E., "Generation of Metaluminous A - type granitoids by low-pressure melting of calc - alkaline granitoids", Geology, vol. 25, pp. 743 - 746, 1997.
[78] Whalen J.B, Jenner G.A, Longstaffe F.J, Robert F. and Gariépy $\mathrm{C}$., "Geochemical and isotopic $(\mathrm{O}, \mathrm{Nd}, \mathrm{Pb}$ and $\mathrm{Sr})$ constraints on A-type granite petrogenesis based on the Topsails Igneous Suite Newfoundland Appalachians", Journal of Petrology, vol. 37, pp. 1463-1489, 1996.

[79] Cullers, R.L., Graf, J.L., "Rare earth elements in igneous rocks of the continental crust: Intermediate and silicic rocks-ore Petrogenesis", In: Henderson, P. (Ed.), Rare Earth Element Geochemistry, Elsevier, pp. 275-316, 1984.

[80] Charoy, B Raimbault L., "Zr, Th and REE-rich biotite differentiates in the A-type granite pluton of Suzhou (eastern China): the key role of Fluorine", Journal of Petrology, vol. 35, pp. 919-962, 1993.

[81] Show, D.M., "A review of K-Rb fractionation trends by covariance analysis", Geochimica et Cosmochimica Acta, vol. 32, pp. 573-601, 1968.

[82] Wilson, M., Igneous Petrogenesis:_A global tectonic approach. Unwin Hyman Ltd. 1989.

[83] Rollinson, H.R., Using Geochemical Data: Evaluation, Presentation, Interpretation. Longman scientific and technical; Copublished in the United States with John Wiley and Sons, inc., New York, 1993.

[84] Leal L. R. B., Wilson T, Cunha J. C, Macambira M. J. B., "Archean tonalitic- trondhjemitic and granitic plutonism in the Gavio Block, San Francisco Craton, Bahia, Brazil: geochemical and geochronological characteristics", Revista Brasileira de Geociências, vol. 28, pp. 209-220, 1998.

[85] Chen B., Jahn B., Wei C., "Petrogenesis of Mesozoic granitoids in the Dabie UHP complex, Central China: trace element and Nd-Sr isotop evidence", Lithos, vol. 60, pp. 67-88, 2002.

[86] Ragland P.C, Basic analytical petrology. Oxford University Press, New York, 1989.

[87] Kochhar, N., "The Malani Supercontinent: Middle East connection during Late Proterozoic", In: Shrivastava, K.L. (Ed.), Economic Mineralization. Scientific Publishers, Jodhpur, pp. 15-25, 2009.

[88] Bonin, B., “A-type granites and related rocks: Evolution of a concept, problems and prospects", Lithos, vol. 97, pp. 1-29, 2007. 